Pacific

Journal of

Mathematics

\title{
FOLD MAPS AND IMMERSIONS FROM THE VIEWPOINT OF COBORDISM
}

BOLDIZSÁR KALMÁR 


\title{
FOLD MAPS AND IMMERSIONS FROM THE VIEWPOINT OF COBORDISM
}

\author{
BOLDIZSÁR KALMÁR
}

\begin{abstract}
We obtain complete geometric invariants of cobordism classes of oriented simple fold maps of $(n+1)$-dimensional manifolds into an $n$-dimensional manifold $N^{n}$ in terms of immersions with prescribed normal bundles. We compute that for $N^{n}=\mathbb{R}^{n}$ the cobordism group of simple fold maps is isomorphic to the direct sum of the $(n-1)$-st stable homotopy group of spheres and the $(n-1)$-st stable homotopy group of the space $\mathbb{R} P^{\infty}$. By using geometric invariants defined in the author's earlier works, we also describe the natural map of the simple fold cobordism group to the fold cobordism group in terms of natural homomorphisms between cobordism groups of immersions. We also compute the ranks of the oriented bordism groups of simple fold maps.
\end{abstract}

\section{Introduction}

Fold maps of $(n+1)$-dimensional manifolds into $n$-dimensional manifolds are smooth singular maps that have the formula

$$
f\left(x_{1}, \ldots, x_{n+1}\right)=\left(x_{1}, \ldots, x_{n-1}, x_{n}^{2} \pm x_{n+1}^{2}\right)
$$

as a local normal form around each singular point. Fold maps can be considered as the natural generalizations of Morse functions. Let $f: Q^{n+1} \rightarrow N^{n}$ be a fold map. The set of singular points of the fold map $f$ is a 2-codimensional smooth submanifold in the source manifold $Q^{n+1}$, and $f$ restricted to its singular points is a 1-codimensional immersion into the target manifold $N^{n}$. This immersion together with more detailed information about the neighborhood of the set of singular points in $Q^{n+1}$ can be used as a geometric invariant (see [Kalmár 2008a] and Section 1.3 here) of fold cobordism classes (see Definition 1.1) of fold maps, and by this way we obtain a geometric relation between fold maps and immersions via cobordisms. In [Kalmár 2008a; 2007b], we defined these invariants for negative codimensional ${ }^{1}$

MSC2000: primary 57R45; secondary 57R90, 57R42, 55Q45.

Keywords: fold singularity, immersion, cobordism, simple fold map, stable homotopy group. The author was supported by Canon Foundation in Europe and has been supported by JSPS.

${ }^{1}$ If we have a map $f: M^{m} \rightarrow P^{p}$ of an $m$-dimensional manifold into a $p$-dimensional manifold, then the codimension of the map $f$ is the integer $p-m$. 
fold maps in full generality, and we used them to detect direct summands of the cobordism groups of negative codimensional fold maps. Here, we study these invariants in the case of -1-codimensional fold maps with some additional restrictions on their singular fibers. (For singular fibers, see [Levine 1985; Saeki 2004]. For cobordism groups of fold maps whose singular fiber components contain only a finite number of points, see [Ando 2001; Sadykov 2005; Saeki 2002].)

Simple fold maps are fold maps with at most one singular point in each connected component of a singular fiber. From this definition it follows that the only possible singular fibers whose singular points have the minus sign in the above normal form are the disjoint unions of a finite number of "figure eight" singular fibers and circle components, provided that the source manifold is orientable or the simple fold map is oriented. ${ }^{2}$ Simple fold maps have been studied, for example, by Levine [1985], Saeki [1992; 1993a; 1996], Sakuma [1994] and Yonebayashi [1999]. The existence of a simple fold map on a manifold gives strong conditions about the structure of the manifold (for example, about the existence of simple fold maps on orientable 3-manifolds; see [Saeki 1996]). If we have a simple fold map of an oriented manifold or an oriented simple fold map, then the immersion of the singular set has trivial normal bundle in the target manifold $N^{n}$, and moreover there is a canonical trivialization corresponding to the number of regular fiber components in a neighborhood of a singular fiber.

The main result of this paper is that our geometric invariants (compare with [Szúcs 2008, Part III]) describe completely the set of cobordism classes of oriented simple fold maps of $(n+1)$-dimensional manifolds into an $n$-dimensional manifold $N^{n}$. By using a Pontryagin-Thom-type construction, we prove that the cobordism classes of oriented simple fold maps of $(n+1)$-dimensional manifolds into an $n$-dimensional manifold $N^{n}$ are in natural bijection with the set of stable homotopy classes of continuous maps of the one point compactification of the manifold $N^{n}$ into the Thom space of the trivial line bundle over the space $\mathbb{R} P^{\infty}$. As a special case, we obtain that the oriented cobordism group of simple fold maps of oriented $(n+1)$-dimensional manifolds into $\mathbb{R}^{n}$ is isomorphic to the $n$-th stable homotopy group of the space $S^{1} \vee S \mathbb{R} P^{\infty}$, where $S \mathbb{R} P^{\infty}$ denotes the suspension of $\mathbb{R} P^{\infty}$.

We also describe the natural homomorphism that maps a simple fold cobordism class to its fold cobordism class in terms of natural homomorphisms between cobordism groups of immersions with prescribed normal bundles. In this way, we obtain results about the "inclusion" of the cobordism group of simple fold maps into that of fold maps. We also obtain the analogous results about bordisms (see Definition 5.1) of fold maps.

${ }^{2}$ We call a fold map $f$ oriented if the kernel of the differential of $\left.f\right|_{R}$ is oriented, where $R$ denotes the set of regular points of $f$. 
The statement giving the main technical tool to prove our results is that if we restrict a fold map $f: Q^{n+1} \rightarrow N^{n}$ to the submanifold $f^{-1}\left(P_{\mathfrak{F}}\right)$, where $P_{\mathfrak{F}}$ denotes a narrow closed tubular neighborhood of the $f$-image of the singular fibers of type $\mathfrak{F}$, then this restriction can be considered as a locally trivial bundle with some map-germ as fiber and with a compact group (which depends only on the singular fiber $\mathfrak{F}$ ) as structure group (Theorems 6.1 and 6.3). In the case of positive codimensional singular maps, such a map-germ would be a (classical) germ around isolated points, while in our case (that is, the negative codimension case) it is a map-germ around a positive dimensional complex. Only the case of germs around isolated points is well understood [Jänich 1978; Wall 1980]. Handling this problem is one of the results of our present paper.

The paper is organized as follows. In Section 1, we give several basic definitions and notations. In Section 2, we state our main results. In Section 3, we prove our main theorems. In Section 4, we give explicit descriptions of the "inclusion" of the simple fold cobordism groups into the fold cobordism groups in low dimensions. In Section 5, we give analogous results about bordism groups of simple fold maps. In Section 6, we prove theorems about the bundle structures of fold maps and symmetries of singular fibers.

Notations. In this paper, we denote by $\amalg$ the disjoint union, by $\gamma^{k}$ the universal $k$-dimensional real vector bundle over $B O(k)$, and by $\epsilon_{X}^{k}$ (or $\left.\epsilon^{k}\right)$ the trivial $k$-plane bundle over the space $X$ (respectively over the point). The symbols $\operatorname{det} \xi^{k}$ and $T \xi^{k}$ denote the determinant line bundle and the Thom space of the $k$-dimensional real vector bundle $\xi^{k}$, respectively. For $k \leq n$, the $\operatorname{symbol} \operatorname{Imm}\left(\xi^{k}, N^{n}\right)$ denotes the cobordism group of $k$-codimensional immersions into an $n$-dimensional manifold $N^{n}$ whose normal bundles are induced from the real vector bundle $\xi^{k}$ (this group is isomorphic to the group $\left\{\dot{N}^{n}, T \xi^{k}\right\}$ [Wells 1966], where $\dot{N}^{n}$ denotes the onepoint compactification of the manifold $N^{n}$ and the symbol $\{X, Y\}$ denotes the group of stable homotopy classes of continuous maps from the space $X$ to the space $Y)$. For $k>n$, the $\operatorname{symbol} \operatorname{Imm}\left(\xi^{k}, N^{n}\right)$ denotes the trivial group. The symbol $\pi_{n}^{s}(X)$ (or $\pi_{n}^{s}$ ) denotes the $n$-th stable homotopy group of the space $X$ (respectively spheres). We denote by $\operatorname{id}_{A}$ the identity map of the space $A$, by $\epsilon$ a small positive real number, and by $\Omega_{n}$ the oriented cobordism group of closed $n$-dimensional manifolds.

\section{Simple fold maps, fold cobordisms and geometric invariants}

Simple fold maps. Let $Q^{n+1}$ and $N^{n}$ be smooth manifolds of dimensions $n+1$ and $n$, respectively. Let $p \in Q^{n+1}$ be a singular point of a smooth map $f: Q^{n+1} \rightarrow N^{n}$. A smooth map $f$ has a fold singularity at the singular point $p$ if we can write $f$ in 
some local coordinates around $p$ and $f(p)$ in the form

$$
f\left(x_{1}, \ldots, x_{n+1}\right)=\left(x_{1}, \ldots, x_{n-1}, x_{n}^{2} \pm x_{n+1}^{2}\right) .
$$

A smooth map $f: Q^{n+1} \rightarrow N^{n}$ is called a fold map if $f$ has only fold singularities.

Singularities with sign + or - in the above normal form are called definite or indefinite fold singularities, respectively.

Let $S_{1}(f)$ and $S_{0}(f)$ denote the set of indefinite and definite fold singularities of $f$ in $Q^{n+1}$, respectively. Let $S_{f}$ denote the set $S_{0}(f) \cup S_{1}(f)$. Note that $S_{f}$ is an $(n-1)$-dimensional submanifold of the manifold $Q^{n+1}$.

If $f: Q^{n+1} \rightarrow N^{n}$ is a fold map in general position, then the map $f$ restricted to the singular set $S_{f}$ is a general position, codimension one immersion into the target manifold $N^{n}$.

Since every fold map is in general position after a small perturbation, and since we study maps under the equivalence relations cobordism and bordism (see Definitions 1.1 and 5.1, respectively), in this paper we can restrict ourselves to studying fold maps that are in general position. A fold map $f$ is in general position unless we say otherwise.

A fold map $f: Q^{n+1} \rightarrow N^{n}$ is called a simple fold map if every connected component of an arbitrary fiber of $f$ contains at most one singular point.

A fold map $f: Q^{n+1} \rightarrow N^{n}$ is called framed if the immersion $\left.f\right|_{S_{1}(f)}$ of its indefinite fold singular set is framed, that is, its normal bundle is trivialized.

A fold map $f: Q^{n+1} \rightarrow N^{n}$ is called oriented if there is a chosen consistent orientation of all fibers at their regular points (for example, in the case of oriented source and target manifolds).

From the definition of simple fold maps it follows that an indefinite singular fiber $^{3}$ of an oriented simple fold map or of a simple fold map of an oriented manifold must be the disjoint union of a finite number of copies of the figure eight and circles.

Note that an oriented simple fold map is framed in a canonical way since the immersion of its indefinite fold singular set has a canonical trivialization corresponding to the number of regular fiber components in a neighborhood of a figure eight singular fiber.

Stein factorization. We use the notion of the Stein factorization of a smooth map $f: Q^{q} \rightarrow N^{n}$, where $Q^{q}$ and $N^{n}$ are smooth manifolds of dimensions $q$ and $n$, respectively, and $q \geq n$. Two points $p_{1}, p_{2} \in Q^{q}$ are equivalent if $p_{1}$ and $p_{2}$ lie on the same component of an $f$-fiber. Let $W_{f}$ denote the quotient space of $Q^{q}$ with respect to this equivalence relation, and let $q_{f}: Q^{q} \rightarrow W_{f}$ be the quotient map.

\footnotetext{
${ }^{3}$ That is, a singular fiber that contains only indefinite fold singular points. For singular fibers, see [Levine 1985; Saeki 2004].
} 


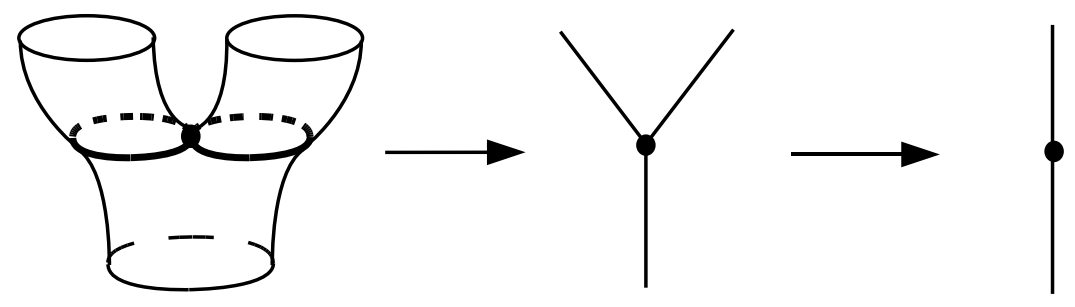

Figure 1. The Stein factorization of a representative of the figure eight singular fiber.

Then there exists a unique continuous map $\bar{f}: W_{f} \rightarrow N^{n}$ such that $f=\bar{f} \circ q_{f}$. The space $W_{f}$ or the factorization of $f$ into the composition of $q_{f}$ and $\bar{f}$ is called the Stein factorization of $f$. We call $\bar{f}$ the Stein factorization of $f$ as well. It is known that if $f$ is a generic smooth map of a closed $q$-dimensional manifold into $N^{n}$ (for example, if $f$ is a fold map in general position), then its Stein factorization $W_{f}$ is a compact $n$-dimensional $\mathrm{CW}$ complex.

For the Stein factorization of a representative of the singular fiber figure eight in the case of $n=1$, see Figure 1.

\section{Cobordisms of simple fold maps.}

Definition 1.1 (cobordism). For $i=0,1$, two oriented fold maps (respectively two oriented simple fold maps) $f_{i}: Q_{i}^{n+1} \rightarrow N^{n}$ from closed $(n+1)$-dimensional manifolds $Q_{i}^{n+1}$ into an $n$-dimensional manifold $N^{n}$ are cobordant (respectively simple cobordant) if

(1) there exists an oriented (respectively oriented simple) fold map $F: X^{n+2} \rightarrow$ $N^{n} \times[0,1]$ from a compact $(n+2)$-dimensional manifold $X^{n+2}$;

(2) $\partial X^{n+2}=Q_{0}^{n+1} \amalg Q_{1}^{n+1}$;

(3) $F$ is equal to $f_{0} \times \operatorname{id}_{[0, \epsilon)}$ on $Q_{0}^{n+1} \times[0, \epsilon)$ and is equal to $f_{1} \times \operatorname{id}_{(1-\epsilon, 1]}$ on $Q_{1}^{n+1} \times(1-\epsilon, 1]$, where $Q_{0}^{n+1} \times[0, \epsilon)$ and $Q_{1}^{n+1} \times(1-\epsilon, 1]$ are small collar neighborhoods of $\partial X^{n+2}$ with the identifications $Q_{0}^{n+1}=Q_{0}^{n+1} \times\{0\}$ and $Q_{1}^{n+1}=Q_{1}^{n+1} \times\{1\}$

(4) the orientations of the fold maps $f_{0}, f_{1}$ and $F$ under the above identifications are consistent.

We then call $F$ a cobordism between $f_{0}$ and $f_{1}$.

This clearly defines an equivalence relation on the set of oriented fold maps (respectively oriented simple fold maps) from closed $(n+1)$-dimensional manifolds to an $n$-dimensional manifold $N^{n}$. In the case of an oriented target manifold $N^{n}$ this cobordism relation coincides with the analogous cobordism relation of (simple) 
fold maps of oriented manifolds, where the manifold $X^{n+2}$ should be oriented and should satisfy $\partial X^{n+2}=Q_{0}^{n+1} U\left(-Q_{1}^{n+1}\right)$.

Let us denote by $\mathscr{C}_{0 b_{\mathfrak{f}}}\left(N^{n}\right)$ the set of all cobordism classes of oriented fold maps into an $n$-dimensional manifold $N^{n}$ and by $\mathscr{C o b}_{\mathfrak{s}}\left(N^{n}\right)$ the set of all simple cobordism classes of oriented simple fold maps into an $n$-dimensional manifold $N^{n}$. We define a commutative semigroup ${ }^{4}$ operation in the usual way on the sets of cobordism classes $\mathscr{C}_{\mathrm{ob}}\left(N^{n}\right)$ and $\mathscr{C}_{\mathrm{ob}}\left(N^{n}\right)$ by the disjoint union, which is a group operation in the case of $N^{n}=\mathbb{R}^{n}$.

Definition 1.2. Two oriented framed fold maps $f_{i}: Q_{i}^{n+1} \rightarrow N^{n}$ (with $i=0,1$ ) are framed cobordant if they are oriented cobordant by an oriented framed fold map $F: X^{n+2} \rightarrow N^{n} \times[0,1]$, in the sense of Definition 1.1, such that the framing of $F$ is compatible with the framings of $f_{0}$ and $f_{1}$.

We denote the framed cobordism classes into an $n$-dimensional manifold $N^{n}$ by $\mathscr{C o b}_{\mathfrak{f}}^{\text {fr }}\left(N^{n}\right)$; this clearly forms a commutative semigroup under the operation of disjoint union.

Bundles of fold germs. We now summarize some properties of the usual germs $\left(\mathbb{R}^{2}, 0\right) \rightarrow(\mathbb{R}, 0)$.

Let us define the indefinite fold germ and the definite fold germ by

$$
\begin{aligned}
g_{\text {indef }}:\left(\mathbb{R}^{2}, 0\right) & \rightarrow(\mathbb{R}, 0), \quad(x, y) \mapsto x^{2}-y^{2}, \\
g_{\text {def }}:\left(\mathbb{R}^{2}, 0\right) & \rightarrow(\mathbb{R}, 0), \quad(x, y) \mapsto x^{2}+y^{2} .
\end{aligned}
$$

We say that a pair $\left(\alpha:\left(\mathbb{R}^{2}, 0\right) \rightarrow\left(\mathbb{R}^{2}, 0\right), \beta:(\mathbb{R}, 0) \rightarrow(\mathbb{R}, 0)\right)$ of diffeomorphism germs is an automorphism of a germ $g:\left(\mathbb{R}^{2}, 0\right) \rightarrow(\mathbb{R}, 0)$ if $g \circ \alpha=\beta \circ g$.

If we have a fold map $f: Q^{n+1} \rightarrow N^{n}$ with nonempty indefinite fold singular set $S_{1}(f)$, then we have the commutative diagram

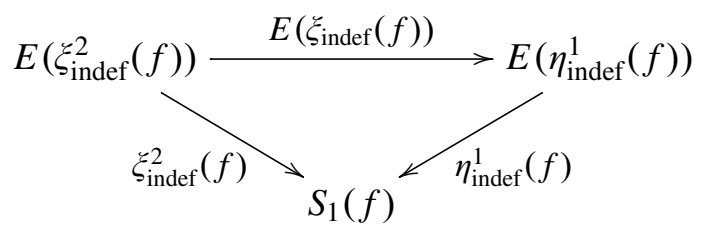

that is, over $S_{1}(f)$ for small positive real numbers $\epsilon$ and $\delta$, we have

(1) a family of small open squares $(-\epsilon, \epsilon) \times(-\epsilon, \epsilon)$ denoted by

$$
\xi_{\text {indef }}^{2}(f): E\left(\xi_{\text {indef }}^{2}(f)\right) \rightarrow S_{1}(f) ;
$$

\footnotetext{
${ }^{4}$ Throughout the paper, we use the word "semigroup" rather than "monoid", even if we have an identity element.
} 
(2) a family of small open intervals $(-\delta, \delta)$ denoted by

$$
\eta_{\text {indef }}^{1}(f): E\left(\eta_{\text {indef }}^{1}(f)\right) \rightarrow S_{1}(f) \text {; }
$$

(3) a fiberwise map $E\left(\xi_{\text {indef }}(f)\right): E\left(\xi_{\text {indef }}^{2}(f)\right) \rightarrow E\left(\eta_{\text {indef }}^{1}(f)\right)$, which is equivalent on each fiber to a representative of $g_{\text {indef }}$,

and we say that we have an indefinite fold germ bundle ${ }^{5}$ over the indefinite fold singular set $S_{1}(f)$, denoted by $\xi_{\text {indef }}(f): E\left(\xi_{\text {indef }}(f)\right) \rightarrow S_{1}(f)$.

The fiber of $\xi_{\text {indef }}(f)$ is a representative of $g_{\text {indef }}$, its base space is $S_{1}(f)$, and its total space is the fiberwise map

$$
E\left(\xi_{\text {indef }}(f)\right): E\left(\xi_{\text {indef }}^{2}(f)\right) \rightarrow E\left(\eta_{\text {indef }}^{1}(f)\right)
$$

between the total spaces of the bundles $\xi_{\text {indef }}^{2}(f)$ and $\eta_{\text {indef }}^{1}(f)$. We call $E\left(\eta_{\text {indef }}^{1}(f)\right)$ the target of the total space $E\left(\xi_{\text {indef }}(f)\right)$ of $\xi_{\text {indef }}(f)$, and we call the bundle $\eta_{\text {indef }}^{1}(f)$ the target of the indefinite fold germ bundle $\xi_{\text {indef }}(f)$.

By [Szúcs 1993], $\xi_{\text {indef }}(f)$ is a locally trivial bundle with fiber $g_{\text {indef }}$ and an appropriate group of automorphisms $\left(\alpha:\left(\mathbb{R}^{2}, 0\right) \rightarrow\left(\mathbb{R}^{2}, 0\right), \beta:(\mathbb{R}, 0) \rightarrow(\mathbb{R}, 0)\right)$ as structure group. The same holds for the definite fold singular set $S_{0}(f)$ and the definite fold germ $g_{\text {def }}(x, y)=x^{2}+y^{2}$.

Moreover, if we have an oriented fold map $f: Q^{n+1} \rightarrow N^{n}$, then the elements of the structure groups of the above indefinite and definite fold germ bundles are automorphisms $\left(\alpha:\left(\mathbb{R}^{2}, 0\right) \rightarrow\left(\mathbb{R}^{2}, 0\right), \beta:(\mathbb{R}, 0) \rightarrow(\mathbb{R}, 0)\right)$ in which the diffeomorphisms $\alpha$ and $\beta$ both preserve or both reverse the orientation, or in other words, they keep fixed a chosen consistent orientation at the regular points of the level curves.

If we have an indefinite fold germ bundle, then by [Jänich 1978; Wall 1980] its structure group can be reduced to a maximal compact subgroup, namely to the dihedral group of order 8 generated by the automorphisms $\left((x, y) \mapsto(x,-y), \operatorname{id}_{\mathbb{R}}\right)$ and $\left((x, y) \mapsto(-y, x),-\mathrm{id}_{\mathbb{R}}\right)$. Furthermore, the automorphisms that keep fixed a chosen consistent orientation of the level curves of the indefinite fold germ form a subgroup $\mathbb{Z}_{2} \oplus \mathbb{Z}_{2}$, which is generated by $\left((x, y) \mapsto(y, x),-\mathrm{id}_{\mathbb{R}}\right)$ and $\left((x, y) \mapsto(-y,-x),-\mathrm{id}_{\mathbb{R}}\right)$. This group has a subgroup $\mathbb{Z}_{2}$ that is generated by the automorphism $\left((x, y) \mapsto(-x,-y), \mathrm{id}_{\mathbb{R}}\right)$.

If we have an oriented simple fold map (or an oriented framed fold map), then the structure group of its indefinite fold germ bundle is reduced to this subgroup $\mathbb{Z}_{2}$, since other elements of $\mathbb{Z}_{2} \oplus \mathbb{Z}_{2}$ cannot be extended to the singular fiber figure eight (respectively do not keep the trivialization of the target of the total space of the indefinite fold germ bundle). Note that in the case of an oriented fold map without

\footnotetext{
${ }^{5}$ Note that the total space $E\left(\xi_{\text {indef }}(f)\right)$ of this bundle is in fact a map, not a set.
} 
any framing in general this structure group can be more complicated than $\mathbb{Z}_{2}$; see, for example, the singular fiber denoted by $\mathrm{II}^{3}$ in [Saeki 2004]. ${ }^{6}$

The automorphism group of the definite fold germ bundle of an oriented fold map can be reduced to one whose elements are of the form $\left((x, y) \rightarrow T(x, y), \operatorname{id}_{\mathbb{R}}\right)$, where $T$ is an element of the group $\mathrm{SO}(2)$.

Now it follows that the targets of the universal oriented indefinite and definite fold germ bundles are the line bundle $\eta_{\text {indef }}^{1}: \operatorname{det}\left(\gamma^{1} \times \gamma^{1}\right) \rightarrow \mathbb{R} P^{\infty} \times \mathbb{R} P^{\infty}$ and the trivial line bundle $\eta_{\mathrm{def}}^{1}: \epsilon_{\mathbb{C} P}^{1} \rightarrow \mathbb{C} P^{\infty}$, respectively. ${ }^{7}$ Similarly, the target of the universal oriented simple (or framed) indefinite fold germ bundle is the trivial line bundle over $\mathbb{R} P^{\infty}$.

The relation between the universal oriented indefinite fold germ bundle and the universal oriented simple (or framed) indefinite fold germ bundle can be seen in the following bundle inclusion, which is induced by the inclusion of the above subgroup $\mathbb{Z}_{2}$ into the automorphism group $\mathbb{Z}_{2} \oplus \mathbb{Z}_{2}$, that is, by the diagonal map $\mathbb{R} P^{\infty} \rightarrow \mathbb{R} P^{\infty} \times \mathbb{R} P^{\infty}$.

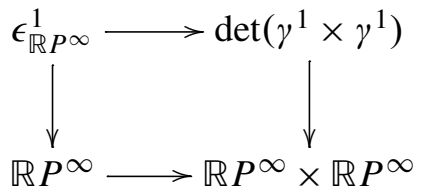

1.3. Cobordism invariants of oriented fold maps. We define the homomorphisms

$$
\begin{aligned}
& \mathscr{I}_{\mathfrak{s}}\left(N^{n}\right): \mathscr{C o b}_{\mathfrak{s}}\left(N^{n}\right) \rightarrow \operatorname{Imm}\left(\epsilon_{\mathbb{R} P}^{1}, N^{n}\right), \\
& \mathscr{D}_{\mathfrak{s}}\left(N^{n}\right): \mathscr{C}_{\mathrm{ob}_{\mathfrak{s}}}\left(N^{n}\right) \rightarrow \operatorname{Imm}\left(\epsilon_{\mathbb{C} P}^{1}, N^{n}\right)
\end{aligned}
$$

by mapping a cobordism class of an oriented simple fold map into the cobordism class of the immersion of its indefinite fold singular set with normal bundle induced from the bundle $\epsilon_{\mathbb{R} P}^{1}$, and into the cobordism class of the immersion of its definite fold singular set with normal bundle induced from the bundle $\epsilon_{\mathbb{C} P^{\infty}}^{1}$, respectively. We define the homomorphisms

$$
\begin{aligned}
& \mathscr{I}_{\mathfrak{f}}\left(N^{n}\right): \mathscr{C o b}_{\mathfrak{f}}\left(N^{n}\right) \rightarrow \operatorname{Imm}\left(\operatorname{det}\left(\gamma^{1} \times \gamma^{1}\right), N^{n}\right), \\
& \mathscr{D}_{\mathfrak{f}}\left(N^{n}\right): \mathscr{C o b}_{\mathfrak{f}}\left(N^{n}\right) \rightarrow \operatorname{Imm}\left(\epsilon_{\mathbb{C} P}^{1}, N^{n}\right)
\end{aligned}
$$

\footnotetext{
${ }^{6}$ We note that it would not be very difficult to extend some of our results to oriented fold maps in an analogous way. The main difference would be that since oriented fold maps can have more complicated singular fibers (for example, the singular fiber denoted by $\mathrm{II}^{3}$ in [Saeki 2004]), the symmetry group of the indefinite fold germ $(x, y) \mapsto x^{2}-y^{2}$ in the case of oriented fold maps is more complicated than in the case of oriented simple fold maps or oriented framed fold maps (where it is generated by the automorphism $(x, y) \mapsto(-x,-y))$.

${ }^{7}$ We use the terminology "universal oriented indefinite and definite fold germ bundles" in the sense that every bundle $\xi_{\text {indef }}(f): E\left(\xi_{\text {indef }}(f)\right) \rightarrow S_{1}(f)$ and $\xi_{\text {def }}(f): E\left(\xi_{\text {def }}(f)\right) \rightarrow S_{0}(f)$ can be induced from them by maps $S_{1}(f) \rightarrow \mathbb{R} P^{\infty} \times \mathbb{R} P^{\infty}$ and $S_{0}(f) \rightarrow \mathbb{C} P^{\infty}$, respectively, uniquely up to homotopy.
} 
by mapping a cobordism class of an oriented fold map into the cobordism class of the immersion of its indefinite fold singular set with normal bundle induced from the bundle $\eta_{\text {indef }}^{1}: \operatorname{det}\left(\gamma^{1} \times \gamma^{1}\right) \rightarrow \mathbb{R} P^{\infty} \times \mathbb{R} P^{\infty}$, and into the cobordism class of the immersion of its definite fold singular set with normal bundle induced from the bundle $\eta_{\mathrm{def}}^{1}: \epsilon_{\mathbb{C} P}^{1} \rightarrow \mathbb{C} P^{\infty}$, respectively; see the previous section.

Analogously, we define the homomorphisms

$$
\begin{aligned}
& \mathscr{F}_{\mathfrak{f}}^{\mathrm{fr}}\left(N^{n}\right): \mathscr{C o b}_{\mathfrak{f}}^{\mathrm{fr}}\left(N^{n}\right) \rightarrow \operatorname{Imm}\left(\epsilon_{\mathbb{R} P \infty}^{1}, N^{n}\right), \\
& \mathscr{D}_{\mathfrak{f}}^{\mathrm{fr}}\left(N^{n}\right): \mathscr{C o b}_{\mathfrak{f}}^{\mathrm{fr}}\left(N^{n}\right) \rightarrow \operatorname{Imm}\left(\epsilon_{\mathbb{C} P}^{1}, N^{n}\right) .
\end{aligned}
$$

Note that the diagram (1-1) induces a homomorphism

$$
\operatorname{Imm}\left(\epsilon_{\mathbb{R} P}^{1}, N^{n}\right) \rightarrow \operatorname{Imm}\left(\operatorname{det}\left(\gamma^{1} \times \gamma^{1}\right), N^{n}\right)
$$

and hence a commutative diagram

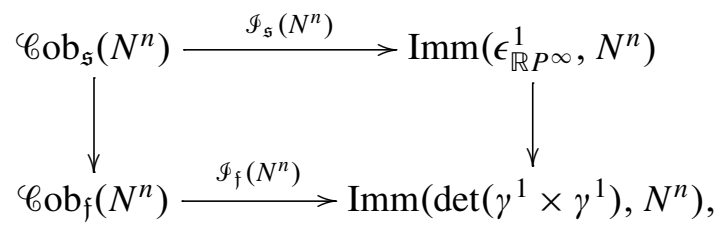

where the left vertical arrow is the natural homomorphism that maps a simple fold cobordism class to its fold cobordism class.

\section{Main results}

Now we are ready to state our main theorems. Let $n \geq 1$.

Theorem 2.1. The semigroup homomorphism $\Phi_{\mathfrak{s}}\left(N^{n}\right)$ is a semigroup isomorphism between the cobordism semigroup $\mathscr{C o b}_{\mathfrak{s}}\left(N^{n}\right)$ of oriented simple fold maps and the group $\operatorname{Imm}\left(\epsilon_{\mathbb{R} P \infty}^{1}, N^{n}\right)$. (The homomorphism $\Phi_{\mathfrak{s}}\left(\mathbb{R}^{n}\right)$ is a group isomorphism.)

Corollary 2.2. The semigroup $\mathscr{C o b}_{\mathfrak{s}}\left(N^{n}\right)$ is a group.

Corollary 2.3. Let $p$ be a prime number. Then

(1) the cobordism group $\mathscr{C o b}_{\mathfrak{s}}\left(N^{n}\right)$ of oriented simple fold maps is isomorphic to the group $\left\{\dot{N}, S^{1}\right\} \oplus\left\{\dot{N}, S \mathbb{R} P^{\infty}\right\}$;

(2) the cobordism group $\mathscr{C o b}_{\mathfrak{s}}\left(\mathbb{R}^{n}\right)$ has no p-torsion if $p>(n+2) / 2$, and its $p$-torsion is $\mathbb{Z}_{p}$ if $p$ is odd and $n=2 p-2$;

(3) the order of an oriented simple fold map is always finite in the fold cobordism group $\mathscr{C o b}_{\mathfrak{f}}\left(\mathbb{R}^{n}\right)(n>1)$. 
Proof. Part (1) follows from the isomorphisms

$$
\begin{aligned}
\operatorname{Cob}_{\mathfrak{s}}\left(N^{n}\right) & \cong \operatorname{Imm}\left(\epsilon_{\mathbb{R} P^{\infty}}^{1}, N^{n}\right), \\
\operatorname{Imm}\left(\epsilon_{\mathbb{R} P}^{1}, N^{n}\right) & \cong\left\{\dot{N}, T \epsilon_{\mathbb{R} P^{\infty}}^{1}\right\} \quad(\text { see [Wells 1966]), } \\
\left\{\dot{N}, T \epsilon_{\mathbb{R} P^{\infty}}^{1}\right\} & \cong\left\{\dot{N}, S^{1} \vee S \mathbb{R} P^{\infty}\right\} \cong\left\{\dot{N}, S^{1}\right\} \oplus\left\{\dot{N}, S \mathbb{R} P^{\infty}\right\} .
\end{aligned}
$$

Parts (2) follows from well-known theorems about the prime-torsions of the groups $\pi_{n-1}^{s}$ and $\pi_{n-1}^{s}\left(\mathbb{R} P^{\infty}\right)$; see [Serre 1951; 1953; Wells 1966, Section III, Theorem 4]. Part (3) follows from the fact that these groups are finite for $n>1$; see the same references.

Let $\phi_{n}^{N}: \mathscr{C o b}_{\mathfrak{s}}\left(N^{n}\right) \rightarrow \mathscr{C o b}_{\mathfrak{f}}\left(N^{n}\right)$ (or $\phi_{n}$ in case $N^{n}=\mathbb{R}^{n}$ ) denote the natural homomorphism that maps a simple fold cobordism class into its fold cobordism class.

Theorem 2.4. (1) The simple fold cobordism group $\mathscr{C o b}_{\mathfrak{s}}\left(N^{n}\right)$ is a direct summand of the framed fold cobordism semigroup $\mathscr{C o b}_{\mathfrak{f}}^{\mathrm{fr}}\left(N^{n}\right)$.

(2) The direct summand $\left\{\dot{N}, S^{1}\right\}$ of $\mathscr{C o b}_{\mathfrak{s}}\left(N^{n}\right)$ is mapped by $\phi_{n}^{N}$ isomorphically onto a direct summand of the fold cobordism semigroup $\mathcal{C o b}_{\mathfrak{f}}\left(N^{n}\right)$.

(3) For $n \geq 2$, if the natural forgetting homomorphism

$$
\operatorname{Imm}\left(\epsilon^{1} \times \gamma^{1}, N^{n}\right) \rightarrow \operatorname{Imm}\left(\gamma^{1} \times \gamma^{1}, N^{n}\right)
$$

induced by the inclusion $\mathbb{R} P^{\infty} \hookrightarrow \mathbb{R} P^{\infty} \times \mathbb{R} P^{\infty}, x \mapsto(*, x)$ is injective, then so is $\phi_{n}^{N}$. The homomorphism $\phi_{1}^{N}$ is injective.

(4) If there exists a fold map from a not null-cobordant $(n+1)$-dimensional manifold to $N^{n}$, then $\phi_{n}^{N}$ is not surjective.

We prove these in Section 3.

By Theorem 2.4, we obtain in Section 4 the following.

Proposition 2.5. For $n=1,2$ the homomorphism $\phi_{n}$ is an isomorphism, $\phi_{3}$ is injective but not surjective, and for $n=5,6$, the homomorphism $\phi_{n}$ is injective.

In this paper, we do not study the homomorphism $\phi_{4}$ and the surjectivity of the homomorphisms $\phi_{5}$ and $\phi_{6}$.

The following proposition will be essential in proving Theorem 2.1.

Proposition 2.6. Every element of the simple fold cobordism group $\mathscr{C o b}_{\mathfrak{s}}\left(N^{n}\right)$ has a representative $g: Q^{n+1} \rightarrow N^{n}$ such that the source manifold $Q^{n+1}$ is the total space $S^{2} \times S_{1}(g)$ of a trivial 2-sphere bundle over the indefinite fold singular set $S_{1}(g)$, and the map $g$ restricted to any fiber $S^{2}$ is a composition $u \circ v$ of an embedding $u: \mathbb{R} \rightarrow N^{n}$ and a Morse function $v: S^{2} \rightarrow \mathbb{R}$ with one indefinite and three definite critical points. 
Corollary 2.7. If there is an oriented simple fold map of an $(n+1)$-dimensional manifold $Q^{n+1}$ into an (oriented) $n$-dimensional manifold, then the manifold $Q^{n+1}$ is (oriented) null-cobordant.

Compare Corollary 2.7 with [Saeki 1992, Proposition 3.12].

Remark 2.8. The surjectivity of the homomorphism $\phi_{2}: \mathscr{C o b}_{\mathfrak{s}}\left(\mathbb{R}^{2}\right) \rightarrow \mathscr{C o b}_{\mathfrak{f}}\left(\mathbb{R}^{2}\right)$ leads to a nice argument showing that every orientable 3-manifold is oriented nullcobordant. Namely, let $M^{3}$ be an orientable 3-manifold. Let $f: M^{3} \rightarrow \mathbb{R}^{2}$ be a smooth stable map. By [Levine 1965], cusp singularities can be eliminated by homotopy (but now elimination by cobordism is enough). Hence, we obtain a fold map $f_{1}: M_{1}^{3} \rightarrow \mathbb{R}^{2}$ whose source manifold is oriented cobordant to $M^{3}$. By the surjectivity of $\phi_{2}$, we have a simple fold map $f_{2}: M_{2}^{3} \rightarrow \mathbb{R}^{2}$ whose source manifold is oriented cobordant to $M^{3}$. Now by Proposition 2.6, $M_{2}^{3}$ is oriented cobordant to a trivial 2-sphere bundle, and therefore the manifold $M^{3}$ is oriented null-cobordant. (Compare with [Costantino and Thurston 2008].)

As a corollary in Case 4.2, we give another proof of the main result of [Kalmár 2007a] together with some geometric invariants, namely:

Theorem 2.9. The oriented cobordism group $\mathscr{C o b}_{\mathfrak{f}}\left(\mathbb{R}^{2}\right)$ offold maps of 3-manifolds into the plane is isomorphic to $\mathbb{Z}_{2} \oplus \mathbb{Z}_{2}$ through the homomorphism $\mathscr{C o b}_{\mathfrak{f}}\left(\mathbb{R}^{2}\right) \rightarrow$ $\operatorname{Imm}\left(\gamma^{1}, \mathbb{R}^{2}\right) \oplus \operatorname{Imm}\left(\gamma^{1}, \mathbb{R}^{2}\right)$ that assigns to a class $[f]$ the $\operatorname{sum}\left[\left.f\right|_{S_{0}(f)}\right] \oplus\left[\left.f\right|_{S_{1}(f)}\right]$ of the immersions of the definite and the indefinite fold singular sets.

Recall that a singular fiber with more than one singular point in a connected component is called nonsimple [Levine 1985].

By the surjectivity of the natural homomorphism $\phi_{2}: \mathscr{C o b}_{\mathfrak{s}}\left(\mathbb{R}^{2}\right) \rightarrow \mathscr{C o b}_{\mathfrak{f}}\left(\mathbb{R}^{2}\right)$, we have the following.

Corollary 2.10. Nonsimple singular fibers of an oriented fold map $f: M^{3} \rightarrow \mathbb{R}^{2}$ can be eliminated by cobordism.

Analogous results for the oriented bordism groups of simple fold maps $\mathscr{B}_{\mathfrak{s}}(n)$ can be found in Section 5. Moreover, we have the following.

Theorem 2.11. The rank of the oriented simple bordism group $\mathscr{B o r}_{\mathfrak{s}}(n)$ is equal to $\operatorname{rank} \Omega_{n}+\sum_{q=0}^{n} \operatorname{rank} \Omega_{q}$.

\section{Proof of main theorems}

Proof of Proposition 2.6. For a given simple fold map $f: Q_{0}^{n+1} \rightarrow N^{n}$, let us construct a fold map $f^{\prime}: Q^{n+1} \rightarrow N^{n}$ by [Kalmár 2007a, Proposition 3.4], and let $g$ denote this fold map $f^{\prime}$. The fold map $g$ is also simple since the construction does not modify the connected components of the singular fibers of $f$ containing only indefinite singular points. Moreover, $f$ and $g$ are simple cobordant. If $f$ is 
oriented, then so is $g$, and $f$ and $g$ are oriented simple cobordant. We can suppose that the indefinite fold singular set $S_{1}(g)$ of $g$ is not empty (otherwise $g$ is nullcobordant as one can see easily: for a precise argument, see [Saeki 1993b]). By the proof of [Kalmár 2007a, Proposition 3.4], the Stein factorization $W_{g}$ of $g$ can be identified with a regular neighborhood of the set $q_{g}\left(S_{1}(g)\right)$. Since $g$ is a simple fold map, the restriction $\left.q_{g}\right|_{S_{1}(g)}$ is an embedding. Because of these facts, we have a surjection $\tilde{g}: W_{g} \rightarrow S_{1}(g) \times[-1,1]$, where $S_{1}(g) \times \mathbb{R}$ is the total space of the trivial normal bundle of the immersion $\left.g\right|_{S_{1}(g)}$. Moreover, the set $\left(\tilde{g} \circ q_{g}\right)^{-1}(V)$, where $V$ is a fiber of the normal bundle of $\left.g\right|_{S_{1}(g)}$, is a 2-dimensional sphere $S^{2}$, and the restriction of $g$ to this sphere is a Morse function with three definite and one indefinite critical points.

Hence the source manifold $Q^{n+1}$ of $g$ is an $S^{2}$ family over $S_{1}(g)$, the map $g$ restricted to any fiber $S^{2}$ is a Morse function with three definite and one indefinite critical points, and $g$ is a composition of a (possibly nontrivial) family $\tilde{g} \circ q_{g}$ : $Q^{n+1} \rightarrow S_{1}(g) \times \mathbb{R}$ of such Morse functions parametrized by the indefinite fold singular set $S_{1}(g)$ and an immersion $S_{1}(g) \times \mathbb{R} \rightarrow N^{n}$ onto a normal neighborhood of $g\left(S_{1}(g)\right)$.

Moreover, it is easy to see that this sphere family is a locally trivial bundle and its structure group can be reduced to $\mathbb{Z}_{2}$ (essentially because of the figure eight subbundle of the sphere bundle coming from the Morse function family; see Corollary 6.4), where the nontrivial element of $\mathbb{Z}_{2}$ acts on the fiber $S^{2}$ as a 180 degree rotation; hence this $S^{2}$-bundle is trivial.

Proof of Theorem 2.1. First, we rephrase our original problem about the cobordism group of simple fold maps. By Proposition 2.6, we can always choose a representative $g$ for an arbitrary simple fold cobordism class in $\mathscr{C}_{\mathrm{ob}}\left(N^{n}\right)$, such that $g$ is equal to the composition

$$
\tilde{S}_{1}(g) \times_{\mathbb{Z}_{2}} S^{2} \stackrel{\varrho}{\longrightarrow} S_{1}(g) \times \mathbb{R} \stackrel{i}{\longrightarrow} N^{n},
$$

where

(1) the map $\varrho: \tilde{S}_{1}(g) \times_{\mathbb{Z}_{2}} S^{2} \rightarrow S_{1}(g) \times \mathbb{R}$ is a family of the Morse function $h: S^{2} \rightarrow \mathbb{R}$ of the 2-sphere $S^{2}$ parametrized by $S_{1}(g)$, where the Morse function $h$ has three definite and one indefinite critical points;

(2) $\mathbb{Z}_{2}$ is the structure group of this family, ${ }^{8}$ where the nontrivial element of $\mathbb{Z}_{2}$ acts on the fiber $h: S^{2} \rightarrow \mathbb{R}$ by a rotation of 180 degrees on $S^{2}$ and identically on $\mathbb{R}$, while keeping fixed the indefinite critical point and one of the definite critical points;

(3) $\tilde{S}_{1}(g)$ denotes the associated principal $\mathbb{Z}_{2}$-bundle over $S_{1}(g)$, and

\footnotetext{
${ }^{8}$ Since this family is a locally trivial bundle, it has a structure group; see Section 6 .
} 
(4) the map $i: S_{1}(g) \times \mathbb{R} \rightarrow N^{n}$ is an immersion onto a normal neighborhood of $g\left(S_{1}(g)\right)$ such that $\left.i\right|_{S_{1}(g) \times\{0\}}$ corresponds to $\left.g\right|_{S_{1}(g)}$.

Moreover, by the same process, a simple fold cobordism $F: X^{n+2} \rightarrow N^{n} \times[0,1]$ between two such representatives $g_{0}$ and $g_{1}$ can be chosen to have a form

$$
\tilde{S}_{1}(F) \times \mathbb{Z}_{2} S^{2} \stackrel{\Upsilon}{\longrightarrow} S_{1}(F) \times \mathbb{R} \stackrel{j}{\longrightarrow} N^{n} \times[0,1]
$$

with the analogous properties, that is, it is a composition of a family $\Upsilon$ of the Morse function $h$ with structure group $\mathbb{Z}_{2}$ parametrized by the indefinite fold singular set of $F$ and an immersion $j$, compatible with $g_{0}$ and $g_{1}$ near the boundary $\partial X^{n+2}$.

Hence, we have a well-defined homomorphism

$$
\mu_{n}\left(N^{n}\right): \mathscr{C o b}_{\mathfrak{s}}\left(N^{n}\right) \rightarrow \operatorname{Imm}\left(\epsilon_{\mathbb{R} P}^{1}, N^{n}\right)
$$

by sending a cobordism class $[g](=[i \circ \varrho])$ to the immersion of $S_{1}(g)$ with normal bundle induced from the trivial line bundle over $\mathbb{R} P^{\infty}\left(=B \mathbb{Z}_{2}\right)$ according to the principal $\mathbb{Z}_{2}$-bundle over $S_{1}(g)$ obtained from the $\mathbb{Z}_{2}$-symmetry of the family $\varrho$.

Proposition 3.1. The homomorphism $\mu_{n}\left(N^{n}\right)$ is an isomorphism.

Proof. First, $\mu_{n}\left(N^{n}\right)$ is surjective because for a given immersion $i: M^{n-1} \rightarrow N^{n}$ with normal bundle $v$ induced from the bundle $\epsilon_{\mathbb{R} P}^{1}$, we can construct a family $\varrho: \widetilde{M}^{n-1} \times_{\mathbb{Z}_{2}} S^{2} \rightarrow M^{n-1} \times \mathbb{R}$ of the Morse function $h: S^{2} \rightarrow \mathbb{R}$, where $\widetilde{M}^{n-1}$ corresponds to the principal $\mathbb{Z}_{2}$-bundle over $M^{n-1}$ obtained from the inducing map $M^{n-1} \rightarrow \mathbb{R} P^{\infty}$ of the normal bundle $v$.

To show $\mu_{n}\left(N^{n}\right)$ is injective, let $g_{i}$ for $i=1,2$ be two oriented simple fold maps. By Proposition 2.6, we can suppose that each $g_{i}$ is given in the form (3-1), that is, $g_{i}$ is essentially a bundle of the Morse function $h$ over $S_{1}\left(g_{i}\right)$, with structure group $\mathbb{Z}_{2}$. If there is a cobordism $j: W^{n} \rightarrow N^{n} \times[0,1]$ between the classes $\mu_{n}\left(N^{n}\right)\left(\left[g_{0}\right]\right)$ and $\mu_{n}\left(N^{n}\right)\left(\left[g_{1}\right]\right)$, then we can construct an $h$-bundle over $W^{n}$ that gives a cobordism between $\tilde{g}_{0}$ and $\tilde{g}_{1}$, where each $\tilde{g}_{i}$ is an oriented simple fold map, obtained from $g_{i}$ by changing orientation on some components. Finally, since there is an orientation-reversing self-diffeomorphism of $S^{2}$ that leaves the Morse function $h$ invariant and that commutes with the $\mathbb{Z}_{2}$-action, we have cobordisms between $g_{0}$ and $\tilde{g}_{0}$ and between $g_{1}$ and $\tilde{g}_{1}$.

The observation that $\mu_{n}\left(N^{n}\right)$ coincides with the homomorphism $\Phi_{\mathfrak{s}}\left(N^{n}\right)$ completes the proof of Theorem 2.1.

A description of the natural homomorphism $\mathscr{C o b}_{\mathfrak{s}}\left(N^{n}\right) \rightarrow \mathscr{C o b}_{\mathfrak{f}}\left(N^{n}\right)$. Let

$$
\phi_{n}^{N}: \mathscr{C o b}_{\mathfrak{s}}\left(N^{n}\right) \rightarrow \mathscr{C o b}_{\mathfrak{f}}\left(N^{n}\right)
$$

( $\phi_{n}$ in the case of $N^{n}=\mathbb{R}^{n}$ ) denote the natural homomorphism that maps a simple fold cobordism class into its fold cobordism class. 
We define the homomorphism

$$
\theta_{\mathfrak{f}}^{N}: \operatorname{Imm}\left(\operatorname{det}\left(\gamma^{1} \times \gamma^{1}\right), N^{n}\right) \rightarrow \operatorname{Imm}\left(\gamma^{1}, N^{n}\right) \oplus \operatorname{Imm}\left(\gamma^{1} \times \gamma^{1}, N^{n}\right)
$$

as follows. Let $\left[h: M^{n-1} \rightarrow N^{n}\right]$ be an element of $\operatorname{Imm}\left(\operatorname{det}\left(\gamma^{1} \times \gamma^{1}\right), N^{n}\right)$. Then the natural forgetting homomorphism $l: \operatorname{Imm}\left(\operatorname{det}\left(\gamma^{1} \times \gamma^{1}\right), N^{n}\right) \rightarrow \operatorname{Imm}\left(\gamma^{1}, N^{n}\right)$ gives a class $\left[h_{1}\right]=l([h])$ in $\operatorname{Imm}\left(\gamma^{1}, N^{n}\right)$. Furthermore, we induce the normal bundle $v(h)$ of the immersion $h$ from the bundle $\operatorname{det}\left(\gamma^{1} \times \gamma^{1}\right) \rightarrow \mathbb{R} P^{\infty} \times \mathbb{R} P^{\infty}$, that is, we have the bundle map

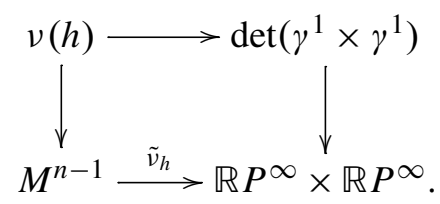

By composing $\tilde{v}_{h}$ with the projection to the first factor $\pi_{1}: \mathbb{R} P^{\infty} \times \mathbb{R} P^{\infty} \rightarrow \mathbb{R} P^{\infty}$, we obtain a line bundle $\lambda^{1}$ over $M^{n-1}$ by the diagram

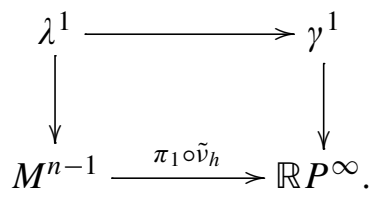

For $n \geq 2$, this line bundle $\lambda^{1}$ over the $(n-1)$-dimensional manifold $M^{n-1}$ gives an (n-2)-dimensional manifold $L^{n-2}$ and an embedding $P_{\lambda^{1}}: L^{n-2} \hookrightarrow M^{n-1}$, which represents the Poincaré dual to the first Stiefel-Whitney class $w_{1}\left(\lambda^{1}\right)$. Hence, we obtain an immersion $h_{2}=h \circ P_{\lambda^{1}}: L^{n-2} \rightarrow N^{n}$ with normal bundle induced from the bundle $\gamma^{1} \times \gamma^{1}$ such that the normal bundle of $h$ corresponds to the first $\gamma^{1}$ factor and the normal bundle of $P_{\lambda^{1}}$ corresponds to the second $\gamma^{1}$ factor, that is, an element of $\operatorname{Imm}\left(\gamma^{1} \times \gamma^{1}, N^{n}\right)$. Now let us define the homomorphism

$$
\theta_{\mathfrak{f}}^{N}: \operatorname{Imm}\left(\operatorname{det}\left(\gamma^{1} \times \gamma^{1}\right), N^{n}\right) \rightarrow \operatorname{Imm}\left(\gamma^{1}, N^{n}\right) \oplus \operatorname{Imm}\left(\gamma^{1} \times \gamma^{1}, N^{n}\right)
$$

by $\theta_{\mathfrak{f}}^{N}([h])=\left[h_{1}\right] \oplus\left[h_{2}\right]$ for $n \geq 2$, and by $\theta_{\mathfrak{f}}^{N}([h])=\left[h_{1}\right]$ for $n=1$ (the group $\operatorname{Imm}\left(\gamma^{1} \times \gamma^{1}, N^{n}\right)$ denotes the trivial group for $n=1$ by our convention).

We define the homomorphism

$$
\theta_{\mathfrak{s}}^{N}: \operatorname{Imm}\left(\epsilon_{\mathbb{R} P}^{1}, N^{n}\right) \rightarrow \operatorname{Imm}\left(\epsilon^{1}, N^{n}\right) \oplus \operatorname{Imm}\left(\epsilon^{1} \times \gamma^{1}, N^{n}\right)
$$

similarly. That is, for $n \geq 1$ and an element $\left[g: M^{n-1} \rightarrow N^{n}\right]$ of the group $\operatorname{Imm}\left(\epsilon_{\mathbb{R} P}^{1}, N^{n}\right)$, define $\left[g_{1}\right]$ to be the image of $[g]$ under the natural forgetting homomorphism $\operatorname{Imm}\left(\epsilon_{\mathbb{R} P}^{1}, N^{n}\right) \rightarrow \operatorname{Imm}\left(\epsilon^{1}, N^{n}\right)$, and for $n \geq 2$ define

$$
\left[g_{2}\right]=\left[g \circ P_{\kappa^{1}}: K^{n-2} \rightarrow N^{n}\right],
$$


where the embedding $P_{\kappa^{1}}: K^{n-2} \hookrightarrow M^{n-1}$ represents the Poincare dual to the first Stiefel-Whitney class of the line bundle $\kappa^{1} \rightarrow M^{n-1}$ obtained from the universal line bundle $\gamma^{1} \rightarrow \mathbb{R} P^{\infty}$ by the map $M^{n-1} \rightarrow \mathbb{R} P^{\infty}$ that induces the normal bundle of the immersion $g: M^{n-1} \rightarrow N^{n}$ from $\epsilon_{\mathbb{R} P \infty}^{1}$.

Let $\gamma_{n}^{N}$ denote the natural forgetting homomorphism

$$
\operatorname{Imm}\left(\epsilon^{1}, N^{n}\right) \oplus \operatorname{Imm}\left(\epsilon^{1} \times \gamma^{1}, N^{n}\right) \rightarrow \operatorname{Imm}\left(\gamma^{1}, N^{n}\right) \oplus \operatorname{Imm}\left(\gamma^{1} \times \gamma^{1}, N^{n}\right)
$$

induced by the inclusion $\mathbb{R} P^{\infty} \hookrightarrow \mathbb{R} P^{\infty} \times \mathbb{R} P^{\infty}, x \mapsto(*, x)$.

By the above constructions and by (1-2), we have the commutative diagram

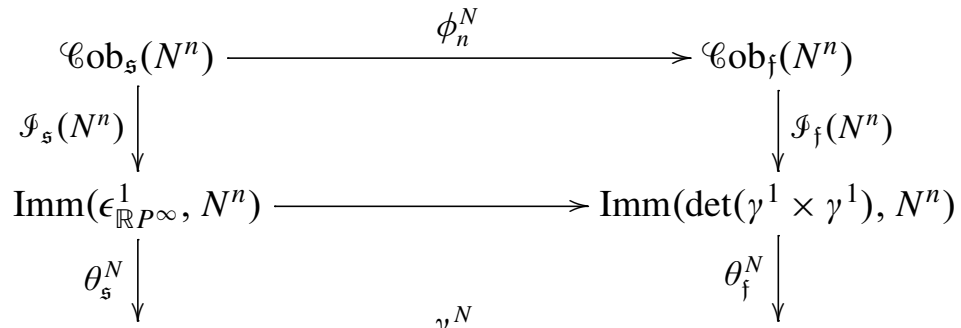

$\operatorname{Imm}\left(\epsilon^{1}, N^{n}\right) \oplus \operatorname{Imm}\left(\epsilon^{1} \times \gamma^{1}, N^{n}\right) \stackrel{\gamma_{n}^{N}}{\longrightarrow} \operatorname{Imm}\left(\gamma^{1}, N^{n}\right) \oplus \operatorname{Imm}\left(\gamma^{1} \times \gamma^{1}, N^{n}\right)$.

Lemma 3.2. The homomorphism $\theta_{\mathfrak{s}}^{N}$ is an isomorphism.

Proof. By the construction of $\theta_{\mathfrak{s}}^{N}$, the statement follows easily. In fact, the homomorphism $\theta_{\mathfrak{s}}^{N}$ yields the isomorphism

$$
\left\{\dot{N}, T \epsilon_{\mathbb{R} P}^{1}\right\} \cong\left\{\dot{N}, S^{1} \vee S \mathbb{R} P^{\infty}\right\} \cong\left\{\dot{N}, S^{1}\right\} \oplus\left\{\dot{N}, S \mathbb{R} P^{\infty}\right\}
$$

under the identifications given by the Pontryagin-Thom construction.

Proof of Theorem 2.4. We define a homomorphism

$$
\psi\left(N^{n}\right): \operatorname{Imm}\left(\epsilon_{\mathbb{R} P}^{1}, N^{n}\right) \rightarrow \mathscr{C o b}_{\mathfrak{f}}^{\mathrm{fr}}\left(N^{n}\right)
$$

so that the composition $\mathscr{I}_{\mathfrak{f}}^{\mathrm{fr}}\left(N^{n}\right) \circ \psi\left(N^{n}\right): \operatorname{Imm}\left(\epsilon_{\mathbb{R} P^{\infty}}^{1}, N^{n}\right) \rightarrow \operatorname{Imm}\left(\epsilon_{\mathbb{R} P}^{1}, N^{n}\right)$ is the identity map, and so that the composition

$$
\operatorname{Imm}\left(\epsilon_{\mathbb{R} P}^{1}, N^{n}\right) \stackrel{\psi\left(N^{n}\right)}{\longrightarrow} \mathscr{C o b}_{\mathfrak{f}}^{\mathrm{fr}}\left(N^{n}\right) \stackrel{\alpha}{\longrightarrow} \mathscr{C o b}_{\mathfrak{f}}\left(N^{n}\right) \stackrel{\mathscr{D}_{\mathfrak{f}}\left(N^{n}\right)}{\longrightarrow} \operatorname{Imm}\left(\epsilon_{\mathbb{C} P}^{1}, N^{n}\right),
$$

in which $\alpha$ is the natural forgetting homomorphism, is an isomorphism of the direct summand $\operatorname{Imm}\left(\epsilon^{1}, N^{n}\right)$ of $\operatorname{Imm}\left(\epsilon_{\mathbb{R} P}^{1}, N^{n}\right)$ onto the direct summand $\operatorname{Imm}\left(\epsilon^{1}, N^{n}\right)$ of $\operatorname{Imm}\left(\epsilon_{\mathbb{C} P}^{1}, N^{n}\right)$. Namely, let $\psi\left(N^{n}\right)$ be the inverse of (3-2), which maps an element in $\operatorname{Imm}\left(\epsilon_{\mathbb{R} P^{\infty}}^{1}, N^{n}\right)$ into the cobordism class of the corresponding representative $g$ (see the proof of Theorem 2.1 and Proposition 3.1). Since (3-2) is an isomorphism by Proposition 3.1, $\psi\left(N^{n}\right)$ is well defined. This gives us Theorem 2.4(1), and also (2) if we observe in the proof of Theorem 2.1 that the three immersions of the definite fold singular set of the representative $g$ (which 
correspond to the three definite critical points of the Morse function $h$ ), where the structure group of the Morse function family is trivial, are parallel to the immersion $\left.g\right|_{S_{1}(g)}$ of the indefinite fold singular set of $g$ and their sum represents the inverse of $\left[\left.g\right|_{S_{1}(g)}\right]$. Because of the diagram (3-3) and since the homomorphisms $\mathscr{I}_{\mathfrak{s}}\left(N^{n}\right)$ and $\theta_{\mathfrak{s}}^{N}$ are isomorphisms, by using (2), we obtain (3). Finally, (4) is obvious from Corollary 2.7 (which we proved independently from Theorem 2.4).

Remark 3.3. The composition

$$
\theta_{\mathfrak{s}}^{N} \circ \mathscr{I}_{\mathfrak{s}}\left(N^{n}\right): \mathscr{C}_{\mathrm{ob}}\left(N^{n}\right) \rightarrow \operatorname{Imm}\left(\epsilon^{1}, N^{n}\right) \oplus \operatorname{Imm}\left(\epsilon^{1} \times \gamma^{1}, N^{n}\right)
$$

gives the isomorphism of Corollary 2.3(1) in a geometric way.

\section{Special cases}

Now, we study in low dimensions the homomorphism $\phi_{n}: \mathscr{C o b}_{\mathfrak{s}}\left(\mathbb{R}^{n}\right) \rightarrow \mathscr{C}_{\mathrm{ob}}\left(\mathbb{R}^{n}\right)$ that maps a simple fold cobordism class into its fold cobordism class, and we prove Proposition 2.5.

Case 4.1 $(n=1)$. By [Ikegami and Saeki 2003], $\mathscr{C o b}_{\mathfrak{f}}(\mathbb{R})$ is isomorphic to $\mathbb{Z}$. By Theorem 2.1, $\mathscr{C o b}_{\mathfrak{s}}(\mathbb{R})$ is isomorphic to $\mathbb{Z}$ (see also [Saeki 2006]), and it is easy to see that the homomorphism $\phi_{1}: \mathscr{C o b}_{\mathfrak{s}}(\mathbb{R}) \rightarrow \mathscr{C}_{\mathrm{ob}}(\mathbb{R})$ is surjective and hence an isomorphism.

Case 4.2 $(n=2)$. By [Kalmár 2007a], $\mathscr{C o b}_{\mathfrak{f}}\left(\mathbb{R}^{2}\right)$ is isomorphic to $\mathbb{Z}_{2} \oplus \mathbb{Z}_{2}$. Here, we give another proof of this isomorphism together with some geometric invariants.

Let $\imath_{2}: \mathscr{C o b}_{\mathfrak{f}}\left(\mathbb{R}^{2}\right) \rightarrow \operatorname{Imm}\left(\gamma^{1}, \mathbb{R}^{2}\right)$ and $\delta_{2}: \mathscr{C o b}_{\mathfrak{f}}\left(\mathbb{R}^{2}\right) \rightarrow \operatorname{Imm}\left(\gamma^{1}, \mathbb{R}^{2}\right)$ denote the homomorphisms that map a fold cobordism class into the immersion of its indefinite and definite fold singular set, respectively.

Now $\pi_{1}^{s} \cong \mathbb{Z}_{2}$ and $\pi_{1}^{s}\left(\mathbb{R} P^{\infty}\right) \cong \mathbb{Z}_{2}$; see [Liulevicius 1963]. By Corollary 2.3(1) and the isomorphisms $\operatorname{Imm}\left(\epsilon^{1}, \mathbb{R}^{2}\right) \cong \pi_{1}^{s}$ and $\operatorname{Imm}\left(\gamma^{1}, \mathbb{R}^{1}\right) \cong \pi_{1}^{s}\left(\mathbb{R} P^{\infty}\right)$, the cobordism group $\mathscr{C o b}_{\mathfrak{s}}\left(\mathbb{R}^{2}\right)$ is isomorphic to $\mathbb{Z}_{2} \oplus \mathbb{Z}_{2}$.

Lemma 4.3. The homomorphism $\left(l_{2} \oplus \delta_{2}\right) \circ \phi_{2}: \mathscr{C o b}_{\mathfrak{s}}\left(\mathbb{R}^{2}\right) \rightarrow \mathbb{Z}_{2} \oplus \mathbb{Z}_{2}$ is an isomorphism.

Proof. It is enough to show that $\left(l_{2} \oplus \delta_{2}\right) \circ \phi_{2}$ is surjective. This can be proved by constructing simple fold maps of oriented 3-manifolds into the plane whose indefinite and definite fold singular sets are immersed into $\mathbb{R}^{2}$ in a prescribed way (see Proposition 2.6).

Lemma 4.4. The homomorphism $\phi_{2}: \mathscr{C o b}_{\mathfrak{s}}\left(\mathbb{R}^{2}\right) \rightarrow \mathscr{C o b}_{\mathfrak{f}}\left(\mathbb{R}^{2}\right)$ is surjective. 
Proof. By the classification of singular fibers of stable fold maps into 3-manifolds [Levine 1985; Saeki 2004], it is easy to show the surjectivity as follows.

Let $\sigma_{\mathfrak{F}}: s_{\mathfrak{F}} \rightarrow(-\epsilon, \epsilon)^{3}$ be a representative of a singular fiber $\mathfrak{F}$. Then the fold map

$$
\left.\sigma_{\mathfrak{F}}\right|_{\sigma_{\mathfrak{F}}^{-1}\left(\partial[-\epsilon / 2, \epsilon / 2]^{3}\right)}: \sigma_{\mathfrak{F}}^{-1}\left(\partial[-\epsilon / 2, \epsilon / 2]^{3}\right) \rightarrow \partial[-\epsilon / 2, \epsilon / 2]^{3}
$$

represents a null-cobordant element in the cobordism group $\mathscr{C o b}_{\mathfrak{f}}\left(\mathbb{R}^{2}\right)$ as follows. For a given fold map $f$ of a 3-manifold into $S^{2}$, by [Kalmár 2007a, Proposition 3.4], we obtain a fold map $f^{\prime}$ cobordant to $f$ such that the Stein factorization $W_{f^{\prime}}$ is contained in a small regular neighborhood of the set $q_{f^{\prime}}\left(S_{1}\left(f^{\prime}\right)\right)$, and the image of $f^{\prime}$ is identified with a small regular neighborhood of $f^{\prime}\left(S_{1}\left(f^{\prime}\right)\right)$, for example, it is contained in $\mathbb{R}^{2} \subset S^{2}$. Moreover it is easy to see that a cobordism of the immersion $\left.f^{\prime}\right|_{S_{1}\left(f^{\prime}\right)}$, which leaves unchanged a neighborhood of the double points, can be extended to a fold cobordism of $f^{\prime}$, which leaves unchanged the indefinite singular fibers of $f^{\prime}$ corresponding to double points.

In this way, we obtain that the singular fiber III $^{4}$ shows a cobordism between a fold map $f_{4}$ with 5 nonsimple singular fibers of type $\mathrm{II}^{2}$ and a simple fold map with 1 singular fiber of type $\mathrm{II}^{1,1}$, and the singular fiber III $^{6}$ shows a cobordism between a fold map $f_{6}$ with 3 nonsimple singular fibers of type $\mathrm{II}^{2}$ and 2 nonsimple singular fibers of type $\mathrm{II}^{3}$ and a fold map with 1 nonsimple singular fiber of type $\mathrm{II}^{3}$ (for the notations of the types of singular fibers, see [Saeki 2004]). Furthermore, $f_{4}$ is cobordant to a fold map with 1 nonsimple singular fiber of type $\mathrm{II}^{2}$, and $f_{6}$ is cobordant to a fold map with 1 nonsimple singular fiber of type $\mathrm{II}^{2}$, as one can see easily.

Hence a fold map that has nonsimple singular fibers is cobordant to a simple fold map.

By the two lemmas above, we can give a proof different from that given in [Kalmár 2007a] for the computation of the cobordism group $\mathscr{C o b}_{\mathfrak{f}}\left(\mathbb{R}^{2}\right)$.

Proof of Theorem 2.9. By Lemma 4.3, $\phi_{2}$ is injective and so by Lemma 4.4 is an isomorphism. Hence the homomorphism

$$
l_{2} \oplus \delta_{2}: \mathscr{C o b}_{\mathfrak{f}}\left(\mathbb{R}^{2}\right) \rightarrow \operatorname{Imm}\left(\gamma^{1}, \mathbb{R}^{2}\right) \oplus \operatorname{Imm}\left(\gamma^{1}, \mathbb{R}^{2}\right)
$$

that maps a fold cobordism class $[f]$ into the direct sum $\left[\left.f\right|_{S_{1}(f)}\right] \oplus\left[\left.f\right|_{S_{0}(f)}\right]$ is also an isomorphism.

Case 4.5 $(n=3)$. By Theorem 2.4, we have to study the natural homomorphism

$$
\gamma_{3,2}: \operatorname{Imm}\left(\epsilon^{1} \times \gamma^{1}, \mathbb{R}^{3}\right) \rightarrow \operatorname{Imm}\left(\gamma^{1} \times \gamma^{1}, \mathbb{R}^{3}\right) .
$$

If $\gamma_{3,2}$ is injective, then so is the homomorphism $\phi_{3}: \mathscr{C o b}_{\mathfrak{s}}\left(\mathbb{R}^{3}\right) \rightarrow \mathscr{C o b}_{\mathfrak{f}}\left(\mathbb{R}^{3}\right)$. 
The group $\operatorname{Imm}\left(\epsilon^{1} \times \gamma^{1}, \mathbb{R}^{3}\right)$ is isomorphic to $\mathbb{Z}_{2}$, and a representative of the generator is an embedded circle whose normal bundle is twisted by 360 degrees as we go once around the circle.

Let $r: \operatorname{Imm}\left(\gamma^{1} \times \gamma^{1}, \mathbb{R}^{3}\right) \rightarrow \operatorname{Imm}\left(\gamma^{1}, \mathbb{R}^{3}\right)$ be the homomorphism such that for an element $[s] \in \operatorname{Imm}\left(\gamma^{1} \times \gamma^{1}, \mathbb{R}^{3}\right)$, an immersed surface representing $r([s])$ is obtained by putting a figure eight in each fiber of the 2-dimensional normal bundle of the representative $s$ in $\mathbb{R}^{3}$, which is invariant under the structure group of the bundle $\gamma^{1} \times \gamma^{1}$.

The map $r \circ \gamma_{3,2}$ is injective because it maps the generator of $\operatorname{Imm}\left(\epsilon^{1} \times \gamma^{1}, \mathbb{R}^{3}\right)$ into the class of an immersed Klein bottle that is not null-cobordant [Pinkall 1985]. By Theorem 2.4, we get that the homomorphism $\phi_{3}$ is injective.

By Theorem 2.4 and the existence of a fold map from a not null-cobordant oriented 4-manifold [Saeki 2004], the homomorphism $\phi_{3}$ is not surjective.

Case 4.6 $(n=5)$. In this case, the homomorphism $\gamma_{5,2}$ is also injective because of the following. By [Hughes 1992; Liulevicius 1963] the forgetting homomorphism $\operatorname{Imm}\left(\epsilon^{1} \times \gamma^{1}, \mathbb{R}^{5}\right) \rightarrow \operatorname{Imm}\left(\gamma^{2}, \mathbb{R}^{5}\right) \operatorname{maps} \operatorname{Imm}\left(\epsilon^{1} \times \gamma^{1}, \mathbb{R}^{5}\right) \cong \operatorname{Imm}\left(\gamma^{1}, \mathbb{R}^{4}\right) \cong \mathbb{Z}_{2}$ injectively into the group $\operatorname{Imm}\left(\gamma^{2}, \mathbb{R}^{5}\right) \cong \mathbb{Z}_{2}$; hence the first forgetting homomorphism in the composition $\operatorname{Imm}\left(\epsilon^{1} \times \gamma^{1}, \mathbb{R}^{5}\right) \rightarrow \operatorname{Imm}\left(\gamma^{1} \times \gamma^{1}, \mathbb{R}^{5}\right) \rightarrow \operatorname{Imm}\left(\gamma^{2}, \mathbb{R}^{5}\right)$ is injective. Therefore the homomorphisms

$$
\gamma_{5,2}: \operatorname{Imm}\left(\epsilon^{1} \times \gamma^{1}, \mathbb{R}^{5}\right) \rightarrow \operatorname{Imm}\left(\gamma^{1} \times \gamma^{1}, \mathbb{R}^{5}\right)
$$

and $\phi_{5}$ are injective.

Case $4.7(n=6)$. The cobordism group $\operatorname{Imm}\left(\epsilon^{1} \times \gamma^{1}, \mathbb{R}^{6}\right) \cong \operatorname{Imm}\left(\gamma^{1}, \mathbb{R}^{5}\right) \cong$ $\pi_{5}^{s}\left(\mathbb{R} P^{\infty}\right)$ vanishes by [Liulevicius 1963]; hence $\phi_{6}$ is clearly injective.

This completes the proof of Proposition 2.5.

\section{Bordisms of fold maps}

Definition 5.1 (bordism). With $i=1,2$, two fold maps (respectively simple fold maps) $f_{i}: Q_{i}^{n+1} \rightarrow N_{i}^{n}$ of closed oriented $(n+1)$-dimensional manifolds $Q_{i}^{n+1}$ into closed oriented $n$-dimensional manifolds $N_{i}^{n}$ are bordant (respectively simple bordant) if

(1) there exists a fold map (respectively simple fold map) $F: X^{n+2} \rightarrow Y^{n+1}$ from a compact oriented $(n+2)$-dimensional manifold $X^{n+2}$ to a compact oriented $(n+1)$-dimensional manifold $Y^{n+1}$;

(2) $\partial X^{n+2}=Q_{0}^{n+1} \amalg\left(-Q_{1}^{n+1}\right)$ and $\partial Y^{n+2}=N_{0}^{n+1} \amalg\left(-N_{1}^{n+1}\right)$; and

(3) $F$ satisfies Definition 1.1(3).

We call the map $F$ a bordism between $f_{0}$ and $f_{1}$. 
We can define a commutative group operation on the set of bordism classes by $\left[f: Q_{1}^{n+1} \rightarrow N_{1}^{n}\right]+\left[g: Q_{2}^{n+1} \rightarrow N_{2}^{n}\right]=\left[f \amalg g: Q_{1}^{n+1} \amalg Q_{2}^{n+1} \rightarrow N_{1}^{n} \amalg N_{2}^{n}\right]$ in the usual way.

We obtain analogous theorems about the bordism group of simple fold maps denoted by $\mathscr{B O r}_{\mathfrak{s}}(n)$, the natural homomorphism $\phi_{n}^{\mathscr{B} o r}: \mathscr{B O r}_{\mathfrak{s}}(n) \rightarrow \mathscr{B}_{\mathfrak{f}}(n)$ into the bordism group of fold maps denoted by $\Re_{0} \mathrm{r}_{\mathfrak{f}}(n)\left(\phi_{n}^{\mathscr{R} \text { or }}\right.$ maps a simple fold bordism class into its fold bordism class), and the framed fold bordism group $\mathscr{B o r}_{\mathfrak{f}}^{\mathrm{fr}}(n)$ defined analogously to the case of cobordism.

Let $\mathscr{B} \operatorname{Imm}\left(\xi^{k}, n\right)$ denote the usual bordism group of $k$-codimensional immersions into closed oriented $n$-dimensional manifolds, whose normal bundles are induced from the vector bundle $\xi^{k}$. Note that this group $\mathscr{I m m}\left(\xi^{k}, n\right)$ is isomorphic to the $n$-th oriented bordism group $\Omega_{n}\left(\Gamma_{\xi^{k}}\right)$ of the classifying space $\Gamma_{\xi^{k}}$ for such immersions, where $\Gamma_{\xi^{k}}$ denotes $\Omega^{\infty} S^{\infty} T \xi^{k}$; see [Schweitzer 1970; Szúcs 1984].

Theorem 5.2. The bordism group $\mathscr{B O r}_{\mathfrak{s}}(n)$ of simple fold maps is isomorphic to $\mathscr{B} \operatorname{Imm}\left(\epsilon^{1}, n\right) \oplus \mathscr{B} \operatorname{Imm}\left(\epsilon^{1} \times \gamma^{1}, n\right)$ for $n \geq 2$ and to $\mathscr{B} \operatorname{Imm}\left(\epsilon^{1}, 1\right)$ for $n=1$.

Proof. Analogously to the homomorphisms $\Phi_{\mathfrak{s}}\left(N^{n}\right)$ and $\theta_{\mathfrak{s}}^{N}$ in the case of cobordism, we have the homomorphisms

$$
\Im_{\mathfrak{s}}^{\mathscr{B} o r}(n): \mathscr{B o r}_{\mathfrak{s}}(n) \rightarrow \mathscr{B} \operatorname{Imm}\left(\epsilon_{\mathbb{R} P}^{1}, n\right)
$$

and

$$
\theta_{\mathfrak{s}}^{\mathscr{B} \text { or }}(n): \mathscr{B} \operatorname{Imm}\left(\epsilon_{\mathbb{R} P}^{1}, n\right) \rightarrow \mathscr{B} \operatorname{Imm}\left(\epsilon^{1}, n\right) \oplus \mathscr{B} \operatorname{Imm}\left(\epsilon^{1} \times \gamma^{1}, n\right),
$$

respectively. Similarly to Theorem $2.1, \mathscr{I}_{\mathfrak{s}}^{\mathscr{B} \text { or }}(n)$ is an isomorphism. The homomorphism $\theta_{\mathfrak{s}}^{\mathscr{乃} o r}(n)$ is surjective because of the following. For classes $\left[h: M^{n-1} \rightarrow N_{1}^{n}\right] \in \mathscr{B} \operatorname{Imm}\left(\epsilon^{1}, n\right) \quad$ and $\quad\left[i: L^{n-2} \rightarrow N_{2}^{n}\right] \in \mathscr{B} \operatorname{Imm}\left(\epsilon^{1} \times \gamma^{1}, n\right)$, by putting the circle $\left(S^{1}, p\right)=\left(\left\{(x+1)^{2}+y^{2}=1\right\},(0,0)\right)$ into each fiber $\mathbb{R}^{2}$ of the normal bundle of the immersion $i$ (here $x$ corresponds to $\epsilon^{1}$ and $y$ corresponds to $\gamma^{1}$ in the fiber), we obtain a framed immersion $j: M^{n-1} \amalg\left(\tilde{L}^{n-2} \times_{\mathbb{Z}_{2}} S^{1}\right) \rightarrow N_{1}^{n} \amalg N_{2}^{n}$, where $\tilde{L}^{n-2}$ is the double cover of $L^{n-2}$ corresponding to the factor $\gamma^{1}$ of the normal bundle of $i$, the restriction $\left.j\right|_{M^{n-1}}$ is equal to $h, \tilde{L}^{n-2} \times_{\mathbb{Z}_{2}}\{p\}$ is identified with $L^{n-2}$, and the restriction $\left.j\right|_{\tilde{L}^{n-2} \times \mathbb{Z}_{2}\{p\}}$ is equal to $i$. Then, a map

$$
\varphi: M^{n-1} \amalg\left(\tilde{L}^{n-2} \times_{\mathbb{Z}_{2}} S^{1}\right) \rightarrow K\left(\mathbb{Z}_{2}, 1\right)
$$

into the Eilenberg-Mac Lane space $K\left(\mathbb{Z}_{2}, 1\right)=\mathbb{R} P^{\infty}$ corresponding to the cohomology class that is the Poincare dual to the homology class represented by the submanifold $L^{n-2}=\tilde{L}^{n-2} \times_{\mathbb{Z}_{2}}\{p\}$ immediately gives a bordism class $[g]$ in $\mathscr{B} \operatorname{Imm}\left(\epsilon_{\mathbb{R} P}^{1}, n\right)$ such that $\theta_{\mathfrak{s}}^{\mathscr{R} o r}(n)([g])=[h] \oplus[i]$. By similar reasons $\theta_{\mathfrak{s}}^{\mathscr{\Re} o r}(n)$ is also injective. For more details, see [Kalmár 2008b, Section 6.1]. 
In other words the bordism group $\mathscr{B O r}_{\mathfrak{s}}(n)$ is isomorphic to the direct sum $\Omega_{n}\left(\Gamma_{\epsilon^{1}}\right) \oplus \Omega_{n}\left(\Gamma_{\epsilon^{1} \times \gamma^{1}}\right)$.

Analogously to Theorem 2.4 in the case of cobordism, we obtain the following.

Theorem 5.3. (1) The simple fold bordism group $\mathscr{B}_{\mathfrak{s}}(n)$ is a direct summand of the framed fold bordism group $\Re_{\mathfrak{f}}^{\mathrm{fr}}(n)$.

(2) The direct summand $\mathscr{B} \operatorname{Imm}\left(\epsilon^{1}, n\right)$ of $\Re_{\mathrm{or}_{\mathfrak{s}}}(n)$ is mapped by $\phi_{n}^{\Re o r}$ isomorphically onto a direct summand of the fold bordism group $\Re_{\mathfrak{f}} \mathrm{r}_{\mathfrak{f}}(n)$.

(3) For $n \geq 2$, if the natural forgetting homomorphism

$$
\mathscr{B} \operatorname{Imm}\left(\epsilon^{1} \times \gamma^{1}, n\right) \rightarrow \mathscr{B} \operatorname{Imm}\left(\gamma^{1} \times \gamma^{1}, n\right)
$$

induced by the inclusion $\mathbb{R} P^{\infty} \hookrightarrow \mathbb{R} P^{\infty} \times \mathbb{R} P^{\infty}, x \mapsto(*, x)$ is injective, then so is $\phi_{n}^{\mathscr{B} o r}$. The homomorphism $\phi_{1}^{\mathscr{B} o r}$ is injective.

(4) If there exists a fold map from a not null-cobordant $(n+1)$-dimensional manifold into a closed oriented $n$-dimensional manifold, then $\phi_{n}^{\text {Bor }}$ is not surjective.

A theorem [Conner and Floyd 1964] that can be applied here says the rank of the bordism groups of any space $X$ can be computed by $\Omega_{*}(X) \otimes \mathbb{Q} \cong H_{*}(X ; \mathbb{Q}) \otimes \Omega_{*}$, that is, $\Omega_{n}(X) \otimes \mathbb{Q} \cong \bigoplus_{p+q=n} H_{p}(X ; \mathbb{Q}) \otimes \Omega_{q}$.

By [Szúcs 1984] the group $\Omega_{n}\left(\Gamma_{\epsilon^{1}}\right) \otimes \mathbb{Q}$ is isomorphic to $\bigoplus_{i=0}^{n} \Omega_{i} \otimes \mathbb{Q}$. By [Wells 1966], the cobordism group $\operatorname{Imm}\left(\epsilon^{1} \times \gamma^{1}, \mathbb{R}^{n}\right) \cong \pi_{n}\left(\Gamma_{\epsilon^{1} \times \gamma^{1}}\right)$ is 2-primary; hence by [Serre 1953] the homology group $H_{r}\left(\Gamma_{\epsilon^{1} \times \gamma^{1}} ; \mathbb{Z}\right)$ is also 2-primary for $r>0$. Therefore $\Omega_{n}\left(\Gamma_{\epsilon^{1} \times \gamma^{1}}\right) \otimes \mathbb{Q}$ is isomorphic to $\Omega_{n} \otimes \mathbb{Q}$, and we have this:

Theorem 5.4. The rank of the simple fold bordism group $\mathscr{B}_{\mathfrak{s}}(n)$ is equal to $\operatorname{rank} \Omega_{n}+\sum_{q=0}^{n} \operatorname{rank} \Omega_{q}$.

Theorem 5.5. The homomorphism $\phi_{n}^{\mathscr{B o r}} \otimes \mathbb{Q}: \mathscr{B}_{\mathfrak{s}}(n) \otimes \mathbb{Q} \rightarrow \mathscr{B o r}_{\mathfrak{f}}(n) \otimes \mathbb{Q}$ is injective.

Proof. The forgetting homomorphism $\Omega_{n}\left(\Gamma_{\epsilon^{1} \times \gamma^{1}}\right) \otimes \mathbb{Q} \rightarrow \Omega_{n}\left(\Gamma_{\gamma^{1} \times \gamma^{1}}\right) \otimes \mathbb{Q}$ induced by the inclusion $\mathbb{R} P^{\infty} \hookrightarrow \mathbb{R} P^{\infty} \times \mathbb{R} P^{\infty}, x \mapsto(*, x)$ is injective because it gives a natural isomorphism from the group $\Omega_{n}\left(\Gamma_{\epsilon^{1} \times \gamma^{1}}\right) \otimes \mathbb{Q} \cong \Omega_{n} \otimes \mathbb{Q}$ into the direct summand $H_{0}\left(\Gamma_{\gamma^{1} \times \gamma^{1}} ; \mathbb{Q}\right) \otimes \Omega_{n} \cong \Omega_{n} \otimes \mathbb{Q}$ of $\Omega_{n}\left(\Gamma_{\gamma^{1} \times \gamma^{1}}\right) \otimes \mathbb{Q}$. By Theorem 5.3, we obtain the statement.

\section{Bundle structure of fold maps}

In Section 3, we used at several steps that a simple fold map $g$ can be considered as a composition of a family of a Morse function with three definite and one indefinite critical points parametrized by the indefinite singular set of $g$, and an immersion. Moreover, this family is locally trivial and its structure group is $\mathbb{Z}_{2}$. We used this description of a simple fold map to assign to $g$ an immersion with normal 
bundle induced from the trivial line bundle $\epsilon_{\mathbb{R} P}^{1}$, which encodes the essential global information about the figure eight singular fibers of $g$. Note that a singular fiber is a map germ (that is, its representatives are maps: for singular fibers, see [Levine 1985; Saeki 2004]). In this section, we deal with the more general situation of fold maps, and the results can be easily applied for simple fold maps as well.

Let $f: Q^{n+1} \rightarrow N^{n}$ be any fold map in general position. Then the map $f$ can be considered as "locally trivial bundles of singular fibers" glued together. More precisely, we have two theorems. The first is an analogue of [Szúcs 1993], while the second is an analogue of the positive codimensional structure group reduction [Jänich 1978; Rimányi and Szúcs 1998; Wall 1980]. We do not give the proofs here, but a detailed proof of Theorem 6.3 can be found in [Kalmár 2008b].

Let $J$ denote the interval $(-\epsilon, \epsilon)$, and let $\sigma_{\mathfrak{F}}: s_{\mathfrak{F}} \rightarrow J^{k}$ be a representative of a singular fiber $\mathfrak{F}$ of $f$. In this paper, we do not distinguish the singular fibers $\mathfrak{F}$ and $\mathfrak{F} \times \operatorname{id}_{\mathbb{R}^{r}}(r>0)$; therefore we suppose that the union of the coordinate hyperplanes in $J^{k}$ is equal to the set $\sigma_{\mathfrak{F}}\left(S_{\sigma_{\mathfrak{F}}}\right)$, and denote the integer $k$ by $\kappa(\mathfrak{F})$. Note that $\kappa(\mathfrak{F})$ is equal to the number of vertices of the graph $\sigma_{\mathfrak{F}}^{-1}(0)$.

Bundle structures of fold maps. Let $g_{1}$ be an autodiffeomorphism of $s_{\mathfrak{F}}$, and let $g_{2}$ be an autodiffeomorphism of $J^{\kappa(\mathfrak{F})}$ such that $g_{2} \circ \sigma_{\mathfrak{F}}=\sigma_{\mathfrak{F}} \circ g_{1}$. We call the pair $\left(g_{1}, g_{2}\right)$ an automorphism of the representative $\sigma_{\mathfrak{F}}$. The automorphism group of $\sigma_{\mathfrak{F}}$ consists of this kind of pairs $\left(g_{1}, g_{2}\right)$. Let $\operatorname{AUT}\left(\sigma_{\mathfrak{F}}\right)$ denote the automorphism group of $\sigma_{\mathfrak{F}} \cdot \operatorname{AUT}\left(\sigma_{\mathfrak{F}}\right)$ is a subgroup of the topological group $\operatorname{DIFF}\left(s_{\mathfrak{F}}\right) \times \operatorname{DIFF}\left(J^{\kappa(\mathfrak{F})}\right)$.

If $\sigma_{\mathfrak{F}}$ is oriented (that is, if the manifold $s_{\mathfrak{F}}$ is oriented), then let $\operatorname{AUT}^{\mathrm{O}}\left(\sigma_{\mathfrak{F}}\right)$ denote the group of automorphisms $\left(g_{1}, g_{2}\right)$ in $\operatorname{AUT}\left(\sigma_{\mathfrak{F}}\right)$ such that $g_{1}$ and $g_{2}$ both preserve or both reverse the orientations of $s_{\mathfrak{F}}$ and $J^{\kappa(\mathfrak{F})}$.

Let $f: Q^{n+1} \rightarrow N^{n}$ be a fold map. For each singular fiber $\mathfrak{F}$ of $f$, let $S_{\mathfrak{F}} \subseteq f\left(S_{f}\right)$ denote the submanifold in $N^{n}$ that is the $f$-image of singular fibers of type $\mathfrak{F}$. Note that $S_{\mathfrak{F}}$ is an $(n-\kappa(\mathfrak{F}))$-dimensional submanifold.

Let $P_{\mathfrak{F}}$ denote the total space of the disk bundle associated with the normal bundle of $S_{\mathfrak{F}}$. The manifold $P_{\mathfrak{F}}$ is embedded into $N^{n}$ in a natural way onto a tubular neighborhood of $S_{\mathfrak{F}}$. Hence, we have the projection $\pi_{\mathfrak{F}}: P_{\mathfrak{F}} \rightarrow S_{\mathfrak{F}}$, and we also have the projection $f^{-1}\left(P_{\mathfrak{F}}\right) \rightarrow S_{\mathfrak{F}}$ defined by the map $\pi_{\mathfrak{F}} \circ f^{\prime}$, where $f^{\prime}=\left.f\right|_{f^{-1}\left(P_{\mathfrak{F}}\right)}$. Therefore, we have the commutative diagram

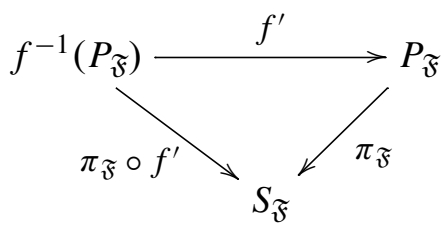

which gives us a kind of bundle denoted by $\xi_{\mathfrak{F}}(f)$ : the total space of $\xi_{\mathfrak{F}}(f)$ is the fiberwise map $f^{\prime}: f^{-1}\left(P_{\mathfrak{F}}\right) \rightarrow P_{\mathfrak{F}}$ between the total spaces of the bundles 
$\pi_{\mathfrak{F}} \circ f^{\prime}: f^{-1}\left(P_{\mathfrak{F}}\right) \rightarrow S_{\mathfrak{F}}$ with fiber $s_{\mathfrak{F}}$ and $\pi_{\mathfrak{F}}: P_{\mathfrak{F}} \rightarrow S_{\mathfrak{F}}$ with fiber $J^{\kappa(\mathfrak{F})}$, the base space of $\xi_{\mathfrak{F}}(f)$ is $S_{\mathfrak{F}}$, and the fiber of $\xi_{\mathfrak{F}}(f)$ is right-left equivalent to the representative $\sigma_{\mathfrak{F}}$.

Then the following theorem can be proved by an argument similar to that in [Szúcs 1993].

Theorem 6.1. The bundle $\xi_{\mathfrak{F}}(f)$ is a locally trivial bundle over $S_{\mathfrak{F}}$ with the representative $\sigma_{\mathfrak{F}}$ as fiber, and with structure group $\operatorname{AUT}\left(\sigma_{\mathfrak{F}}\right)$. If $\sigma_{\mathfrak{F}}$ is oriented, then the structure group is reduced to $\operatorname{AUT}^{\mathrm{O}}\left(\sigma_{\mathfrak{F}}\right)$.

Definition 6.2. Let us suppose that the singular fiber $\mathfrak{F}$ has no circle components and does not contain definite singular points. Let $\pi_{i}: J^{\kappa(\mathfrak{F})} \rightarrow(-\epsilon, \epsilon)$ be the projection to the $i$-th factor for $i=1, \ldots, \kappa(\mathfrak{F})$.

Let us define a Riemannian metric $\varrho$ on the manifold $s_{\mathfrak{F}}$, which is invariant under the action of the maximal compact subgroup of the automorphism group of the multigerm

$$
\sigma_{\mathfrak{F}}:\left(s_{\mathfrak{F}},\left\{p_{i}\right\}_{i=1}^{\kappa(\mathfrak{F})}\right) \rightarrow\left(J^{\kappa(\mathfrak{F})}, 0\right),
$$

where $p_{1}, \ldots, p_{\kappa(\mathfrak{F})}$ are the singular points of $\sigma_{\mathfrak{F}}$ [Jänich 1978; Wall 1980]. Let $F_{i}$ be the subset in $s_{\mathfrak{F}}$ that is the closure of the union of those integral curves of the gradient vector field of the function $\pi_{i} \circ \sigma_{\mathfrak{F}}$ that converge to a singular point of the function $\pi_{i} \circ \sigma_{\mathfrak{F}}$. Let

$$
\tilde{s}_{\mathfrak{F}}=s_{\mathfrak{F}}-\bigcup_{i=1}^{\kappa(\mathfrak{F})} F_{i} \quad \text { and } \quad \tilde{\sigma}_{\mathfrak{F}}=\left.\sigma_{\mathfrak{F}}\right|_{\tilde{S}_{\mathfrak{F}}} .
$$

Then the preimage $\tilde{\sigma}_{\mathfrak{F}}^{-1}(x)$ is diffeomorphic to $\amalg_{2 \kappa(\mathfrak{F})}(-1 / 2,1 / 2)$ for any $x \in J^{\kappa(\mathfrak{F})}$. We can suppose that the $\varrho$-length of each component of each fiber $\tilde{\sigma}_{\mathfrak{F}}^{-1}(x)$ is equal to 1 for $x \in J^{\kappa(\mathfrak{F})}$.

Let $\operatorname{ISO}^{\mathrm{O}}\left(\sigma_{\mathfrak{F}}\right)$ (or $\operatorname{ISO}\left(\sigma_{\mathfrak{F}}\right)$ ) denote the subgroup of $\operatorname{AUT}^{\mathrm{O}}\left(\sigma_{\mathfrak{F}}\right)$ (respectively $\left.\operatorname{AUT}\left(\sigma_{\mathfrak{F}}\right)\right)$ generated by those elements that act on the target $(-\epsilon, \epsilon)^{\kappa(\mathfrak{F})}$ of $\sigma_{\mathfrak{F}}$ by permutations of the factors and reflections with respect to the coordinate hyperplanes and preserve the set $\tilde{s}_{\mathfrak{F}}$ and the Riemannian metrics on the fibers $\tilde{\sigma}_{\mathfrak{F}}^{-1}(x)$ for $x \in J^{\kappa(\mathfrak{F})}$.

$\operatorname{ISO}\left(\sigma_{\mathfrak{F}}\right)$ (respectively $\operatorname{ISO}^{\mathrm{O}}\left(\sigma_{\mathfrak{F}}\right)$ ) is called the symmetry group of $\sigma_{\mathfrak{F}}$.

We can prove the following; see also [Kalmár 2008c].

Theorem 6.3. Let us suppose that the singular fiber $\mathfrak{F}$ has no circle components and does not contain definite singular points. Then, the structure group of the bundle $\xi_{\mathfrak{F}}(f)$ can be reduced to the group $\operatorname{ISO}\left(\sigma_{\mathfrak{F}}\right)\left(\right.$ or $\operatorname{ISO}^{\mathrm{O}}\left(\sigma_{\mathfrak{F}}\right)$ if $f$ is oriented $)$.

By combining the results of Propositions 6.7 and 6.8 of the next section and Theorem 6.3, we obtain the following.

Corollary 6.4. Let $\sigma: s \rightarrow(-\epsilon, \epsilon)$ be a representative of the figure eight singular fiber (see Figure 1). Then the group $\operatorname{ISO}^{\mathrm{O}}(\sigma)$ is isomorphic to $\mathbb{Z}_{2}$. The generator 
of $\mathbb{Z}_{2}$ acts on the figure eight $\sigma^{-1}(0)$ as a 180 degree rotation around the singular point by interchanging the two arcs and preserving their orientations, acts on the source $s$ by interchanging the two upper parts of the "upside-down pants" (see Figure 1$)$, and acts on the target $(-\epsilon, \epsilon)$ identically.

6.5. Symmetry groups of singular fibers. Recall from Definition 6.2 the singular fiber $\mathfrak{F}$.

Definition 6.6. Let $v$ be a vertex of the graph $\sigma_{\mathfrak{F}}^{-1}(0)$. Let $\gamma$ be a small arc in $J^{\kappa(\mathfrak{F})}$ intersecting the hyperplane corresponding to $v$ transversely and only at the origin. Let $U_{v}$ be a small open disk around $v$ in $\sigma_{\mathfrak{F}}^{-1}(\gamma)$. We obtain four edges $a_{1}, \ldots, a_{4}$ with common vertex $v$ in $U_{v} \bigcap \sigma_{\mathfrak{F}}^{-1}(0)$ and a cyclic order $\left(a_{i(1)}, a_{i(2)}, a_{i(3)}, a_{i(4)}\right)$ (where $i$ is a permutation of the set $\{1,2,3,4\}$ ) by considering the locations of the four edges in the disk $U_{v}$. We say that the edges $a_{i(1)}$ and $a_{i(3)}$ are opposite, and $a_{i(1)}$ is the opposite of $a_{i(3)}$, and likewise for $a_{i(2)}$ and $a_{i(4)}$. We say that an autohomeomorphism $h$ of the graph $\sigma_{\mathfrak{F}}^{-1}(0)$ is regular if the $h$-image of opposite edges are always opposite.

For an (oriented) representative $\sigma_{\mathfrak{F}}: s_{\mathfrak{F}} \rightarrow J^{\kappa(\mathfrak{F})}$ of $\mathfrak{F}$, let $\operatorname{ISO}\left(\sigma_{\mathfrak{F}}^{-1}(0)\right)$ denote the group of regular autohomeomorphisms of the graph $\sigma_{\mathfrak{F}}^{-1}(0)$, up to isotopy (that is, two regular autohomeomorphisms are equivalent if they are isotopic); likewise let $\operatorname{ISO}^{\mathrm{O}}\left(\sigma_{\mathfrak{F}}^{-1}(0)\right)$ denote the regular autohomeomorphisms of $\sigma_{\mathfrak{F}}^{-1}(0)$ whose elements preserve the orientation of the fibers of $\sigma_{\mathfrak{F}}$ at their regular points, up to isotopy.

Clearly, we have homomorphisms

$$
\chi^{\mathrm{O}}: \operatorname{ISO}^{\mathrm{O}}\left(\sigma_{\mathfrak{F}}\right) \rightarrow \operatorname{ISO}^{\mathrm{O}}\left(\sigma_{\mathfrak{F}}^{-1}(0)\right) \quad \text { and } \quad \chi: \operatorname{ISO}\left(\sigma_{\mathfrak{F}}\right) \rightarrow \operatorname{ISO}\left(\sigma_{\mathfrak{F}}^{-1}(0)\right)
$$

by restricting an automorphism of $\sigma_{\mathfrak{F}}$ to the graph $\sigma_{\mathfrak{F}}^{-1}(0)$.

Proposition 6.7. The homomorphisms $\chi^{\mathrm{O}}$ and $\chi$ are isomorphisms.

Proof. The union of the coordinate hyperplanes divides $J^{\kappa(\mathfrak{F})}$ into $2^{\kappa(\mathfrak{F})}$ regions, and each region corresponds to a set of "smoothing operations" for the vertices of $\sigma_{\mathfrak{F}}^{-1}(0)$. Therefore, a regular autohomeomorphism $\alpha$ in $\operatorname{ISO}^{\mathrm{O}}\left(\sigma_{\mathfrak{F}}^{-1}(0)\right)$ induces an autohomeomorphism $h$ of $J^{\kappa(\mathfrak{F})}$ that belongs to the group generated by the permutations of the factors and reflections with respect to the coordinate hyperplanes. It is easy to show that $\alpha$ also induces an autohomeomorphism $\tilde{h}$ of $s_{\mathfrak{F}}$ such that $h \circ \sigma_{\mathfrak{F}}=\sigma_{\mathfrak{F}} \circ \tilde{h}$ and such that $\tilde{h}$ restricted to $\sigma_{\mathfrak{F}}^{-1}(0)$ equals $\alpha$. Therefore $\chi^{\mathrm{O}}$ is surjective.

The homomorphism $\chi^{\mathrm{O}}$ is injective because any $g$ in $\operatorname{ISO}^{\mathrm{O}}\left(\sigma_{\mathfrak{F}}\right)$ that satisfies $\chi^{\mathrm{O}}(g)=\mathrm{id}_{\sigma_{\mathfrak{F}}^{-1}(0)}$ fixes setwise each coordinate hyperplane, since $g$ fixes pointwise the vertices of the graph $\sigma_{\mathfrak{F}}^{-1}(0)$.

This $g$ fixes the coordinate hyperplanes pointwise as well: As one can see by looking at the automorphisms of $\left.\sigma_{\mathfrak{F}}\right|_{U_{V}}$, where $U_{V}$ denotes the union of small balls 
in $s_{\mathfrak{F}}$ around the vertices of $\sigma_{\mathfrak{F}}^{-1}(0), g$ fixes the edges pointwise, and therefore it fixes the coordinate axes in the target $J^{\kappa(\mathfrak{F})}$ pointwise. Hence it fixes all of the target $J^{\kappa(\mathfrak{F})}$ pointwise.

If an automorphism of the indefinite fold germ $\left(\mathbb{R}^{2}, 0\right) \ni(x, y) \mapsto x^{2}-y^{2} \in(\mathbb{R}, 0)$ fixes the set $\{x= \pm y\}$ and the target $(\mathbb{R}, 0)$ pointwise, then it is isotopic to the identity. Since $g$ fixes the edges of the graph $\sigma_{\mathfrak{F}}^{-1}(0)$, we obtain that $g$ is the identity in a neighborhood of the indefinite fold singular set of $\sigma_{\mathfrak{F}}$. Hence $g$ is the identity of $\sigma_{\mathfrak{F}}$.

The proof for $\chi$ is analogous.

Proposition 6.8. The groups $\operatorname{ISO}^{\mathrm{O}}\left(\sigma_{\mathfrak{F}}\right)$ and $\operatorname{ISO}\left(\sigma_{\mathfrak{F}}\right)$ are finite, and their orders divide the numbers $\kappa(\mathfrak{F}) ! 4^{\kappa(\mathfrak{F})}$ and $\kappa(\mathfrak{F}) ! 8^{\kappa(\mathfrak{F})}$, respectively.

Proof. Let $H\left(\sigma_{\mathfrak{F}}^{-1}(0)\right)$ be the normal subgroup of $\operatorname{ISO}^{\mathrm{O}}\left(\sigma_{\mathfrak{F}}^{-1}(0)\right)$ consisting of those elements that keep the vertices fixed pointwise. Then the group

$$
\operatorname{ISO}^{\mathrm{O}}\left(\sigma_{\mathfrak{F}}^{-1}(0)\right) / H\left(\sigma_{\mathfrak{F}}^{-1}(0)\right)
$$

is a subgroup of the permutation group of degree $\kappa(\mathfrak{F})$. Moreover, $H\left(\sigma_{\mathfrak{F}}^{-1}(0)\right)$ is a subgroup of the group $\left(\mathbb{Z}_{2}^{2}\right)^{\kappa(\mathfrak{F})}$. Hence the order of $\operatorname{ISO}^{\mathrm{O}}\left(\sigma_{\mathfrak{F}}^{-1}(0)\right)$ divides $\kappa(\mathfrak{F}) ! 4^{\kappa(\mathfrak{F})}$. The proof for $\operatorname{ISO}\left(\sigma_{\mathfrak{F}}\right)$ is analogous, but we have to take the dihedral group of order eight instead of $\mathbb{Z}_{2}^{2}$.

\section{Acknowledgments}

The author thanks Professor András Szúcs for uncountable discussions and suggestions, and the referee, whose comments improved the paper.

\section{References}

[Ando 2001] Y. Ando, "Fold-maps and the space of base point preserving maps of spheres", J. Math. Kyoto Univ. 41:4 (2001), 693-737. MR 2003a:57060 Zbl 1008.57022

[Conner and Floyd 1964] P. E. Conner and E. E. Floyd, Differentiable periodic maps, Ergebnisse der Mathematik und ihrer Grenzgebiete 33, Academic, New York, 1964. MR 31 \#750 Zbl 0125.40103 [Costantino and Thurston 2008] F. Costantino and D. Thurston, "3-manifolds efficiently bound 4manifolds", J. Topol. 1:3 (2008), 703-745. MR 2417451

[Hughes 1992] J. F. Hughes, "Bordism and regular homotopy of low-dimensional immersions", Pacific J. Math. 156:1 (1992), 155-184. MR 93j:57019 Zbl 0768.57014

[Ikegami and Saeki 2003] K. Ikegami and O. Saeki, "Cobordism group of Morse functions on surfaces”, J. Math. Soc. Japan 55:4 (2003), 1081-1094. MR 2004g:57046 Zbl 1046.57020

[Jänich 1978] K. Jänich, "Symmetry properties of singularities of $C^{\infty}$-functions”, Math. Ann. 238:2 (1978), 147-156. MR 80k:58021 Zbl 0384.58011

[Kalmár 2007a] B. Kalmár, "Cobordism group of fold maps of oriented 3-manifolds into the plane", Acta Math. Hungar. 117:1-2 (2007), 1-25. MR 2008h:57044 Zbl 05355852 
[Kalmár 2007b] B. Kalmár, "Fold cobordisms and stable homotopy groups", 2007. Preprint, to appear in Studia Scient. Math. Hungar. arXiv 0704.3147

[Kalmár 2008a] B. Kalmár, "Cobordism invariants of fold maps", pp. 103-115 in Real and complex singularities (São Carlos, Brazil), edited by M. J. Saia and J. Seade, Contemporary Mathematics 459, American Mathematical Society, Providence, RI, 2008. Zbl 05348143

[Kalmár 2008b] B. Kalmár, "Fold maps and immersions from the viewpoint of cobordism", preprint, 2008. arXiv 0705.2731v2

[Kalmár 2008c] B. Kalmár, "Pontryagin-Thom-Szűcs type construction for non-positive codimensional singular maps with prescribed singular fibers", pp. 66-79 in The second Japanese-Australian Workshop on Real and Complex Singularities, RIMS Kôkyûroku 1610, Research Institute for Mathematical Sciences, Kyoto University, 2008.

[Levine 1965] H. I. Levine, “Elimination of cusps”, Topology 3:suppl. 2 (1965), 263-296. MR 31 \#756 Zbl 0146.20001

[Levine 1985] H. Levine, Classifying immersions into $\mathbf{R}^{4}$ over stable maps of 3-manifolds into $\mathbf{R}^{2}$, Lecture Notes in Mathematics 1157, Springer, Berlin, 1985. MR 88f:57056 Zbl 0567.57001

[Liulevicius 1963] A. Liulevicius, "A theorem in homological algebra and stable homotopy of projective spaces”, Trans. Amer. Math. Soc. 109 (1963), 540-552. MR 27 \#6270 Zbl 0134.19202

[Pinkall 1985] U. Pinkall, "Regular homotopy classes of immersed surfaces", Topology 24:4 (1985), 421-434. MR 87e:57028 Zbl 0583.57020

[Rimányi and Szúcs 1998] R. Rimányi and A. Szúcs, "Pontrjagin-Thom-type construction for maps with singularities”, Topology 37:6 (1998), 1177-1191. MR 99g:57031 Zbl 0924.57035

[Sadykov 2005] R. Sadykov, "Bordism groups of special generic mappings", Proc. Amer. Math. Soc. 133:3 (2005), 931-936. MR 2006a:55007 Zbl 1060.57023

[Saeki 1992] O. Saeki, "Notes on the topology of folds", J. Math. Soc. Japan 44:3 (1992), 551-566. MR 93f:57037 Zbl 0764.57017

[Saeki 1993a] O. Saeki, "Simple stable maps of 3-manifolds into surfaces, II", J. Fac. Sci. Univ. Tokyo Sect. IA Math. 40:1 (1993), 73-124. MR 94k:57044 Zbl 0794.57014

[Saeki 1993b] O. Saeki, “Topology of special generic maps into $\mathbf{R}^{3}$ ”, Mat. Contemp. 5 (1993), 161186. MR 95k:57037 Zbl 0855.57026

[Saeki 1996] O. Saeki, "Simple stable maps of 3-manifolds into surfaces”, Topology 35:3 (1996), 671-698. MR 97m:57047 Zbl 0864.57028

[Saeki 2002] O. Saeki, "Cobordism groups of special generic functions and groups of homotopy spheres”, Japan. J. Math. (N.S.) 28:2 (2002), 287-297. MR 2003k:57035 Zbl 1017.57010

[Saeki 2004] O. Saeki, Topology of singular fibers of differentiable maps, Lecture Notes in Mathematics 1854, Springer, Berlin, 2004. MR 2005m:58085 Zbl 1072.57023

[Saeki 2006] O. Saeki, "Cobordism of Morse functions on surfaces, the universal complex of singular fibers and their application to map germs", Algebr. Geom. Topol. 6 (2006), 539-572. MR 2007i: 58052 Zbl 1098.57017

[Sakuma 1994] K. Sakuma, "On the topology of simple fold maps", Tokyo J. Math. 17:1 (1994), 21-31. MR 95h:57034 Zbl 0833.57017

[Schweitzer 1970] P. A. Schweitzer, "Joint cobordism of immersions", pp. 267-282 in The Steenrod algebra and its applications: A conference to celebrate N. E. Steenrod's sixtieth birthday (Columbus, OH 1970), edited by F. P. Peterson, Lecture Notes in Mathematics 168, Springer, Berlin, 1970. MR 43 \#4052 Zbl 0205.53503 
[Serre 1951] J.-P. Serre, "Homologie singulière des espaces fibrés: Applications", Ann. of Math. (2) 54 (1951), 425-505. MR 13,574g Zbl 0045.26003

[Serre 1953] J.-P. Serre, “Groupes d'homotopie et classes de groupes abéliens”, Ann. of Math. (2) 58 (1953), 258-294. MR 15,548c Zbl 0052.19303

[Szúcs 1984] A. Szúcs, "Cobordism groups of immersions with restricted self-intersection", Osaka J. Math. 21:1 (1984), 71-80. MR 85h:57035 Zbl 0553.57012

[Szű́cs 1993] A. Szúcs, "Universal singular map", pp. 491-500 in Topology: Theory and applications, II (Pécs, 1989), edited by Á. Császár, Colloq. Math. Soc. János Bolyai 55, North-Holland, Amsterdam, 1993. MR 94i:58027 Zbl 0828.57023

[Szúcs 2008] A. Szúcs, “Cobordism of singular maps”, Geom. Topol. 12 (2008), 2379-2452.

[Wall 1980] C. T. C. Wall, "A second note on symmetry of singularities", Bull. London Math. Soc. 12:5 (1980), 347-354. MR 81j:58022 Zbl 0424.58006

[Wells 1966] R. Wells, “Cobordism groups of immersions”, Topology 5 (1966), 281-294. MR 33 \#4946 Zbl 0145.20202

[Yonebayashi 1999] Y. Yonebayashi, "Note on simple stable maps of 3-manifolds into surfaces", Osaka J. Math. 36:3 (1999), 685-709. MR 2001a:57043 Zbl 0955.57025

Received May 23, 2008. Revised October 3, 2008.

\section{BOLDIZSÁR KALMÁR}

FACULTY OF MATHEMATICS

KYUSHU UNIVERSITY

6-10-1 HAKOZAKI, HIGASHI-KU

FUKUOKA 812-8581

JAPAN

kalmbold@cs.elte.hu 